\title{
The Effects of Dietary Nitrate Supplementation on Explosive Exercise Performance: A Systematic Review
}

\author{
Rachel Tan ${ }^{1}\left(\mathbb{D}\right.$, Leire Cano $^{2}$, Ángel Lago-Rodríguez ${ }^{3, *}$ and Raúl Domínguez ${ }^{4,5}$ (i) \\ 1 Faculty of Sports Medicine, Natural Sciences Division, Pepperdine University, Malibu, CA 90263, USA; \\ rachel.tan@pepperdine.edu \\ 2 Independent Researcher, 48991 Getxo, Spain; leire_140@hotmail.com \\ 3 Movement, Brain and Health Group, Center of Higher Education Alberta Giménez, \\ 07013 Palma de Mallorca, Spain \\ 4 Departamento de Motricidad Humana y Rendimiento, Universidad de Sevilla, 41013 Sevilla, Spain; \\ rdherrera@us.es \\ 5 Studies Research Group in Neuromuscular Responses (GEPREN), University of Lavras, \\ Lavras 37200-000, Brazil \\ * Correspondence: angellagorodriguez@gmail.com; Tel.: +34-680-330-105
}

check for

updates

Citation: Tan, R.; Cano, L.;

Lago-Rodríguez, Á.; Domínguez, R.

The Effects of Dietary Nitrate

Supplementation on Explosive

Exercise Performance: A Systematic

Review. Int. J. Environ. Res. Public

Health 2022, 19, 762. https://

doi.org/10.3390/ijerph19020762

Academic Editor: Richard B.

Kreider

Received: 29 September 2021

Accepted: 4 January 2022

Published: 11 January 2022

Publisher's Note: MDPI stays neutral with regard to jurisdictional claims in published maps and institutional affiliations.

Copyright: (C) 2022 by the authors. Licensee MDPI, Basel, Switzerland. This article is an open access article distributed under the terms and conditions of the Creative Commons Attribution (CC BY) license (https:// creativecommons.org/licenses/by/ $4.0 /)$.

\begin{abstract}
Dietary nitrate supplementation is evidenced to induce physiological effects on skeletal muscle function in fast-twitch muscle fibers and may enhance high-intensity exercise performance. An important component of sport-specific skills is the ability to perform explosive movements; however, it is unclear if nitrate supplementation can impact explosive efforts. We examined the existing evidence to determine whether nitrate supplementation improves explosive efforts lasting $\leq 6 \mathrm{~s}$. PubMed, Scopus and Directory of Open Access Journals (DOAJ) were searched for articles using the following search strategy: (nitrate OR nitrite OR beetroot) AND (supplement OR supplementation) AND (explosive OR power OR high intensity OR high-intensity OR sprint* OR "athletic performance"). Out of 810 studies, 18 were eligible according to inclusion criteria. Results showed that 4 of the 10 sprint-type studies observed improved sprint time, power output, and total work in cycling or running, whereas 4 of the 10 resistance-based exercise studies observed improvements to power and velocity of free-weight bench press as well as isokinetic knee extension and flexion at certain angular velocities. These results suggest that nitrate potentially improves explosive exercise performance, but further work is required to clarify the factors influencing the efficacy of nitrate in different exercise modalities.
\end{abstract}

Keywords: beetroot juice; ergogenic aid; power; sports nutrition; muscle; nitric oxide

\section{Introduction}

The ability to generate power (i.e., the product of force and velocity) during explosive movements is a key determinant for performance in various sports [1]. Explosive movements can be characterized by efforts that last $\leq 6 \mathrm{~s}$ as the main energetic contribution is derived from the phosphocreatine system [2]. Maximal power production is linked to factors such as the rate of force development (i.e., velocity of contraction) [3], strength [4], and the recruitment of type II muscle fibers [5], and thus the manipulation of such variables, through exercise or dietary interventions, may improve explosive efforts [6,7].

The International Olympic Committee (IOC) recently classified five supplements to have a strong evidence base for enhancing high-intensity exercise performance, which includes caffeine, beta-alanine, sodium bicarbonate, beetroot juice, and creatine [8]. Out of these aforementioned supplements, dietary nitrate $\left(\mathrm{NO}_{3}{ }^{-}\right)$, administered most often as concentrated beetroot juice, is the only supplement from a natural food source [9], and the notion that this simple, vegetable-derived strategy can improve athletic performance has intrigued scientists and athletes alike [10]. Dietary $\mathrm{NO}_{3}{ }^{-}$can be obtained from the 
diet through consuming green leafy vegetables, which have $\sim 4 \mathrm{mmol}$ of $\mathrm{NO}_{3}{ }^{-}$per $100 \mathrm{~g}$ of produce [9]; however, beetroot juice supplements (one to two doses of concentrated shots, each containing $70 \mathrm{~mL} \times \sim 6.4 \mathrm{mmol}$ of $\mathrm{NO}_{3}{ }^{-}$) would be more practical compared to ingesting nitrate-rich meals before competition or training to minimize gastrointestinal discomfort. To date, based on the available evidence, a minimum dose of at least $\sim 6 \mathrm{mmol}$ of $\mathrm{NO}_{3}{ }^{-}$, ingested within 2-3 h prior to the start of exercise, is required to induce physiological effects [11]. Although high amounts of $\mathrm{NO}_{3}{ }^{-}$have been traditionally viewed to have detrimental physiological effects, diets rich in $\mathrm{NO}_{3}{ }^{-}$content from fruits and vegetables have reported cardioprotective effects, suggesting that $\mathrm{NO}_{3}{ }^{-}$sourced from vegetables confer benefits that outweigh traditional perceived risks [12,13]. Furthermore, to our knowledge, the highest dose of $\mathrm{NO}_{3}{ }^{-}$investigated thus far is a dose of $\sim 19.5 \mathrm{mmol}$ of $\mathrm{NO}_{3}{ }^{-}$, which was tolerated by elite athletes [14].

The effects of dietary $\mathrm{NO}_{3}{ }^{-}$ingestion are attributed to the elevation in nitric oxide (NO), a signaling molecule that regulates vascular, metabolic, and contractile processes [15], through the absorption and metabolism of $\mathrm{NO}_{3}{ }^{-}$in the enterosalivary pathway [16]. Following consumption, exogenous $\mathrm{NO}_{3}{ }^{-}$is absorbed by the gastrointestinal tract and enters the systemic circulation [16]. Upon reaching the salivary glands, $\mathrm{NO}_{3}{ }^{-}$re-enters the oral cavity via protein transporters (e.g., sialin) and is concentrated in the saliva [16]. Consequently, anaerobic facultative bacteria that reside in the dorsal part of the tongue utilize salivary $\mathrm{NO}_{3}{ }^{-}$for bacterial respiration [17] and reduce $\sim 20 \%$ of $\mathrm{NO}_{3}{ }^{-}$into nitrite $\left(\mathrm{NO}_{2}{ }^{-}\right.$) (Figure 1). A portion of swallowed $\mathrm{NO}_{2}{ }^{-}$is reduced into nitric oxide (NO) in the stomach [18], but the remaining $\mathrm{NO}_{2}{ }^{-}$is transported to organs and tissues for storage or conversion into $\mathrm{NO}$ and S-nitrosothiols [19] via various $\mathrm{NO}_{2}{ }^{-}$reducing proteins such as deoxyhemoglobin, deoxymyoglobin, and xanthine oxidoreductase [15]. Thus, it is commonly accepted that increased $\mathrm{NO}$ following dietary $\mathrm{NO}_{3}{ }^{-}$ingestion is evidenced by a rise in plasma $\left[\mathrm{NO}_{2}{ }^{-}\right]$([] denote concentration), the precursor molecule to $\mathrm{NO}$ [11]. Interestingly, this $\mathrm{NO}_{3}{ }^{-}{ }^{-} \mathrm{NO}_{2}{ }^{-}-\mathrm{NO}$ pathway prioritizes yielding $\mathrm{NO}$ in areas undergoing acidosis [20] or hypoxia [21] and, therefore, the areas that stand to benefit the most from NO. For example, contracting skeletal muscle during exercise exhibits these environments, providing a rationale to implement $\mathrm{NO}_{3}{ }^{-}$supplementation strategies during exercise [22-27].

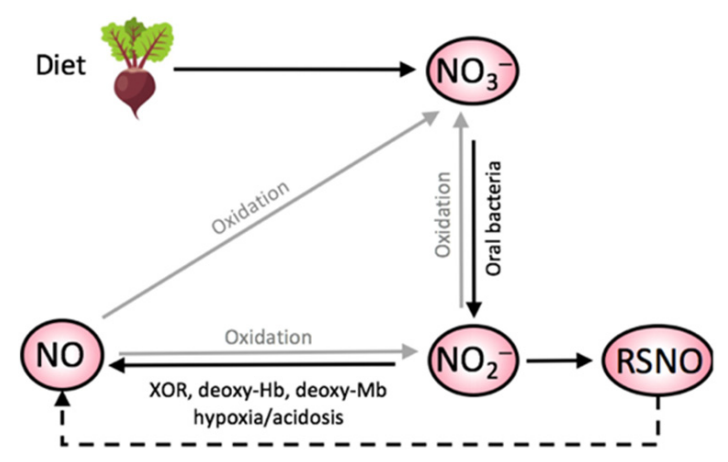

Figure 1. A schematic diagram of the nitrate-nitrite-nitric oxide $\left(\mathrm{NO}_{3}{ }^{-}-\mathrm{NO}_{2}{ }^{-}-\mathrm{NO}\right)$ pathway, which is facilitated by environments of low oxygen tension and acidosis. The conversion of $\mathrm{NO}_{2}{ }^{-}$to NO occurs via various enzymatic reactions; S-nitrosothiols (RSNO) can decompose to form NO. $\mathrm{XOR}=$ xanthine oxidoreductase; deoxy- $\mathrm{Hb}$ = deoxyhemoglobin; deoxy-Mb = deoxymyoglobin.

Dietary $\mathrm{NO}_{3}{ }^{-}$has various proposed physiological mechanisms that may exert ergogenic effects, which include improved contractile function [28,29] c.f. [30], improved energy cost of force production [31], better homogenous blood flow distribution [32], and more pronounced effects in type II muscle fibers [28,32-34]. However, specific to power production, $\mathrm{NO}_{3}{ }^{-}$supplementation may improve skeletal muscle force production and function by modulating calcium release [28,29] and/or sensitivity [35], particularly favoring fatigue-sensitive type II muscle fibers [28], although it should be noted that alterations to calcium-handling proteins have not been observed in humans $[29,30]$. The exact mechanism 
by which $\mathrm{NO}_{3}{ }^{-}$may evoke structural changes to proteins regulating excitation-contraction coupling remains elusive but could be due to nitrosylation or molecular signaling via cyclic guanosine monophosphate [35]. Taken together, the mechanistic bases of dietary $\mathrm{NO}_{3}{ }^{-}$ supplementation support its efficacy in various exercise modalities and may suggest that exercise relying on high-velocity or high-power contractions, and thus a greater relative proportion of type II muscle fibers, would be improved following $\mathrm{NO}_{3}{ }^{-}$supplementation.

The current body of literature has evidenced that $\mathrm{NO}_{3}{ }^{-}$can cause exercise performance enhancements in intermittent high-intensity exercise bouts [23,26], endurance exercise [27], and in some forms of resistance exercise [24], although it is acknowledged that discrepancies between studies exist [26,36]. For example, power output has been shown to improve with $\mathrm{NO}_{3}{ }^{-}$in isokinetic dynamometry [37-40], as well as in cycling [41-44] and time to reach peak power $[41-43,45]$. More recently, a single dose of $\mathrm{NO}_{3}{ }^{-}$improved the mean power $\left(\mathrm{P}_{\text {mean }}\right)$ and mean velocity $\left(\mathrm{V}_{\text {mean }}\right)$ of bench press by $\sim 19 \%$ and $\sim 7 \%$, respectively [46]; however, there was no effect of $\mathrm{NO}_{3}{ }^{-}$ingestion on bench press in another study [47]. Interestingly, performance has been reported to improve following $\mathrm{NO}_{3}{ }^{-}$ingestion during exercise protocols likely recruiting a greater proportion of type II fibers [48], such as cycling at faster pedaling rates compared to slower pedaling rates $[33,34]$ and isokinetic knee extensions at high angular velocities [37], reinforcing the possibility that $\mathrm{NO}_{3}{ }^{-}$favors impacting high-velocity contractions. Furthermore, $\mathrm{NO}_{3}{ }^{-}$-induced improvements may predominate during the initial phases of contraction, as evidenced when power output was improved during shorter (6 s) maximal sprints compared to longer (30 s) maximal sprints [49]. In support of this notion, a recent meta-analysis by Wong et al. [36] interpreted that the peak power output $\left(\mathrm{P}_{\text {peak }}\right)$ of single maximum sprinting efforts was more likely to be improved, but not during repeated maximum sprints, suggesting that the benefits of dietary $\mathrm{NO}_{3}{ }^{-}$may be conferred better to contractions at earlier phases of force production. Together, the evidence supports the potential for dietary $\mathrm{NO}_{3}{ }^{-}$in impacting powerful contractions with high velocities during short explosive efforts, but it is currently unclear how $\mathrm{NO}_{3}{ }^{-}$may influence these movements, such as explosive efforts, emphasizing that further research is required.

The aim of this review was to perform a summary of investigations that have investigated the effect of $\mathrm{NO}_{3}{ }^{-}$supplementation in exercise involving explosive efforts $(\leq 6 \mathrm{~s})$ and to explore the factors that might have a role in the efficacy of $\mathrm{NO}_{3}{ }^{-}$for explosive movements during various exercise modalities.

\section{Materials and Methods}

\subsection{Literature Search}

The present review conducted a systematic search using the Preferred Reporting Items for Systematic Reviews and Meta-Analysis (PRISMA) guidelines [50] and was conducted for studies that investigated $\mathrm{NO}_{3}{ }^{-}$supplementation on explosive exercise performance using PubMed, Scopus, and Directory of Open Access Journals (DOAJ) with no filter based on date of publication. The following terms were used: (nitrate OR nitrite OR beetroot) (concept 1) AND (supplement OR supplementation) (concept 2) AND (explosive OR power OR high intensity OR high-intensity OR sprint* OR “athletic performance") (concept 3). A total of 18 articles met the eligibility criteria according to the Population, Intervention, Comparison, Outcomes, and Study (PICOS) framework (Table 1) for this systematic review. Two researchers independently checked all the search results and performed the screening and eligibility of studies. After contrasting outcomes between these two researchers, results showing discrepancy were evaluated by a third researcher. All documents that were not related with $\mathrm{NO}_{3}{ }^{-}$supplementation or were not associated with the established PICOS criteria were discarded. 
Table 1. Inclusion criteria according to the Population, Intervention, Comparison, Outcomes, and Study (PICOS) framework.

\begin{tabular}{cc}
\hline Parameter & Inclusion Criteria \\
\hline Population & Adult healthy population \\
Intervention & Acute and/or chronic Supplementation with $\mathrm{NO}_{3}{ }^{-}$ \\
Comparison & A placebo condition (supplementation depleted on $\left.\mathrm{NO}_{3}{ }^{-}\right)$ \\
Outcome & Variables related to performance of explosive efforts $(\leq 6 \mathrm{~s}[2])$ \\
Setting & Randomized double-blind placebo-controlled studies \\
\hline
\end{tabular}

\subsection{Quality Assessment}

A quality assessment was performed by two authors (L.C., R.D.) using the PEDro scale [51]. Following previous works [52], a 12th item was added to the scale: "Did the study assess the effectiveness of the blinding to the nitrate supplementation?", in an attempt to adapt to the assessment of methodological quality and the specific characteristics of the studies included in this systematic review. Questions were answered with "yes" if the criteria were satisfied or with "no" if the criteria were not satisfied. Since PEDro's first item is not included in the final score, the maximal score possible for one study was 11. Based on final scores, studies were classified as showing excellent (10-11 points), good (7-9 points), fair $(5-6$ points), or poor ( $<5$ points) methodological quality [ 53,54$]$. Each study's quality assessment was independently performed by two authors (L.C. and R.D.). Thereafter, these two researchers discussed and resolved by consensus any discrepancy, taking into consideration the opinion of a third author if no consensus was reached (Á.L.-R.).

\subsection{Data Extraction}

Two authors (L.C. and R.D.) independently extracted data from each study using a predefined data sheet, and they further discussed and resolved by consensus any potential discrepancy afterwards. The following information was compiled from each study: cite, information related to the sample (sample size, sex, level of physical fitness), supplementation protocol, exercise protocol, and performance outcomes. When a significant effect of $\mathrm{NO}_{3}{ }^{-}$supplementation was reported $(p \leq 0.05)$, outcomes were presented according to the increase observed after supplementation, compared to placebo condition (expressed as \%), which was calculated using the next equation: (mean value after supplementation condition-mean value after placebo condition)/mean value after placebo condition $\times 100$. In addition, Cohen's $d$ and 95\% confidence intervals are included in the results. When values for Cohen's $d$ calculations were not reported in the original article, it was calculated based on the available data. When data were unavailable for calculation, Cohen's $d$ was reported as 'unknown'. Effect sizes were defined as trivial $(<0.2)$, small $(<0.5)$, moderate $(<0.8)$, and large $(>0.8)$ [55].

\section{Results}

\subsection{Study Selection}

A total of 810 results were initially obtained, which were reduced to 428 results after excluding duplicates $(n=382)$. After excluding articles that were not related to the topic or did not comply with the type of document of interest of the present study $(n=253)$, a total of 175 manuscripts were originally identified for this review. After screening for inclusion criteria, 157 articles were excluded since they did not evaluate healthy participants $(n=25)$, did not test a control group $(n=1)$, presented methodological errors $(n=1)$, did not use $\mathrm{NO}_{3}{ }^{-}$supplementation $(n=10)$, and / or did not assess voluntary explosive efforts $(n=120)$. A total of 18 articles were finally selected for this systematic review (Figure 2). Of the 18 studies selected, 2 studies $[56,57]$ had exercise protocols that included both sprint and resistance explosive efforts and, thus, were divided into both Tables 2 and 3 for the corresponding type of exercise. 


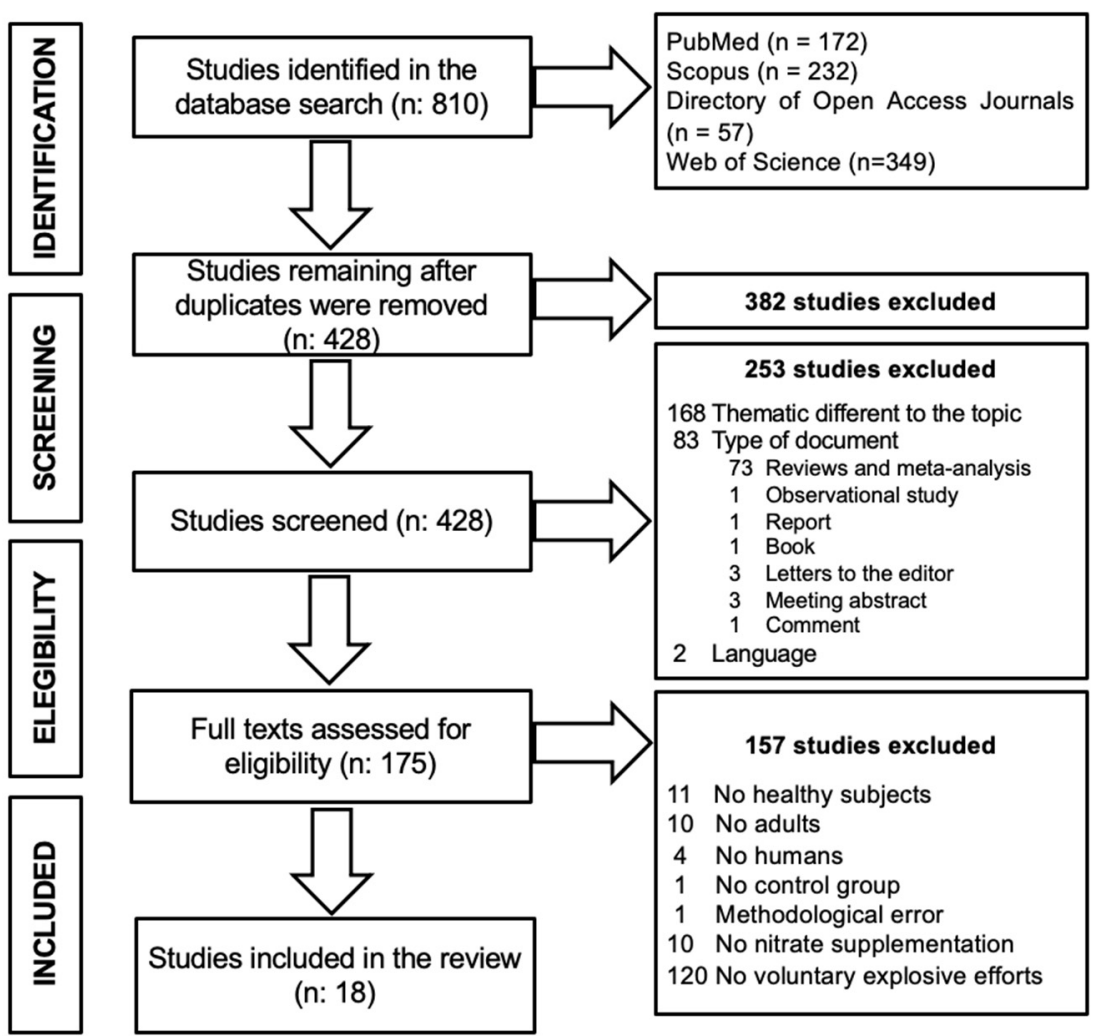

Figure 2. Preferred Reporting Items for Systematic Reviews and Meta-Analysis (PRISMA) flowchart [58].

The experimental design and results of all reviewed studies in this article are provided in Tables 2 and 3, while the quality of assessments is provided in Table 4. The exercise modalities used to assess explosive exercise performance included sprint- and/or resistancebased exercise efforts that were $\leq 6 \mathrm{~s}$ [2]. Recovery periods ranged between $16 \mathrm{~s}$ [59] and $2 \min [44]$.

A total of 18 studies were selected for this systematic review (Figure 2), which evaluated a total sample of 267 participants. Only 20 females were tested, with the remaining 247 being males. Out of these 267 participants, 228 were moderately active [37,40,46,49,57,59-67], 13 were elite athletes [44], and 26 did not practice any sport $[56,68]$. A total of eight studies assessed the effects of $\mathrm{NO}_{3}{ }^{-}$supplementation in explosive resistance efforts [37,40,46,47,65-68], whereas another eight evaluated explosive sprint-type efforts [44,49,59-64], with the remaining two studies performing a combination of both tests [56,57].

\subsection{Study Characteristics}

Sprint-based exercises were performed using cycle ergometers [44,49,59,62,63], running lanes [60], a tennis court [57], and a treadmill [56], with locations such as an indoor gymnasium [61] or in the laboratory [64]. Buck et al. [61] and Thompson et al. [59] performed a team sport simulation test. López-Samanes et al. [57] assessed the speed of tennis serves and evaluated an agility test. The remaining studies $[56,57,60,64]$ employed sprints, without other types of exercise protocols. Among the sprints performed on a cycle ergometer, two were tested under specific environmental conditions, such as hypoxia [63] or elevated temperature and humidity [62]. 
Table 2. Description of studies that have investigated the effects of dietary $\mathrm{NO}_{3}{ }^{-}$supplementation on high-intensity, sprint-type explosive efforts in humans. There were 4 studies out of 10 that observed significant performance improvements to sprints in cycling [44,49,59] and running [60]. Specifically, $\mathrm{NO}_{3}{ }^{-}$improved cycling $\mathrm{P}_{\max }$ [44], $\mathrm{RPM}_{\mathrm{opt}}$ [44], $\mathrm{P}_{\text {mean }}$ [49], and total work performed [59].

\begin{tabular}{|c|c|c|c|c|}
\hline Reference & Subjects & Supplementation & Exercise Protocol & Results \\
\hline Buck et al. [61] & $\begin{array}{l}13 \text { female amateur } \\
\text { basketball and } \\
\text { soccer players }\end{array}$ & $\begin{array}{l}6 \mathrm{~d} \text { of } \mathrm{BR} \text { supplementation } \\
\left(\mathrm{NO}_{3}{ }^{-} 6 \mathrm{mmol} \text { per day }\right)\end{array}$ & $\begin{array}{l}\text { Before, during, and after } \\
60 \text { min in a simulated } \\
\text { team-game: } 6 \times 20 \mathrm{~m} \\
\text { running sprints, } 25 \mathrm{~s} \text { rest }\end{array}$ & $\begin{array}{l}\leftrightarrow \text { Best sprint time (set } 1):-0.3 \%(3.68 \pm 0.26 \text { vs. } 3.69 \pm 0.25 \mathrm{~s} ; \mathrm{d}=0.04[-0.85 \text { to } 0.77]) \\
\leftrightarrow \text { Best sprint time (set }):-0.8 \%(3.77 \pm 0.32 \text { vs. } 3.80 \pm 0.25 \mathrm{~s} ; \mathrm{d}=0.11[-0.91 \text { to } 0.71]) \\
\leftrightarrow \text { Best sprint time (set } 3):+1.1 \%(3.81 \pm 0.32 \text { vs. } 3.77 \pm 0.25 \mathrm{~s} ; \mathrm{d}=0.14[-0.68 \text { to } 0.95])\end{array}$ \\
\hline Rimer et al. [44] & $\begin{array}{c}13 \text { competitive athletes } \\
\text { (female, } n=2 \text {, male, } n=11 \text { ) }\end{array}$ & $\begin{array}{l}2.5 \mathrm{~h} \text { prior to exercise acute } \\
\text { BR ingestion } \\
\left(\mathrm{NO}_{3}^{-}-11.2 \mathrm{mmol}\right)\end{array}$ & $\begin{array}{l}4 \times 3 \text { to } 4 \text { s cycling sprints, } \\
2 \text { min rest }\end{array}$ & $\begin{array}{l}\uparrow \text { Pmax: }+6 \pm 2.6 \text { vs. } 2 \pm 3.8 \%(\mathrm{~d}=1.21[0.31 \text { to } 2.07]) \\
\uparrow \text { RPMopt: }+6.5 \pm 11.4 \text { vs. } 0.3 \pm 4.1 \%(\mathrm{~d}=0.79[-0.14 \text { to } 1.54])\end{array}$ \\
\hline Smith et al. [62] & $\begin{array}{l}12 \text { male recreationally } \\
\text { active athletes }\end{array}$ & $\begin{array}{l}3 \mathrm{~h} \text { prior to exercise acute } \\
\mathrm{BR} \text { ingestion } \\
\left(\mathrm{NO}_{3}^{-} 6.2 \mathrm{mmol}\right)\end{array}$ & $\begin{array}{l}20 \times 6 \text { s cycling sprints in } \\
\text { temperate }\left(22.5^{\circ} \mathrm{C}\right) \text { and } \\
\text { hot environmental } \\
\text { conditions }\left(30^{\circ} \mathrm{C}\right) \\
114 \mathrm{~s} \text { rest }\end{array}$ & $\begin{array}{l}\leftrightarrow \text { Ppeak (hot): }-6.0 \%(659 \pm 100 \text { vs. } 683 \pm 139 \mathrm{~W} ; \mathrm{d}=0.21[-1.04 \text { to } 0.66]) \\
\leftrightarrow \text { Pmean }(\text { temperate }):-1.6 \%(562 \pm 120 \text { vs. } 571 \pm 124 \mathrm{~W} ; \mathrm{d}=0.08[-0.92 \text { to } 0.78]) \\
\leftrightarrow \text { Pmean (hot): }-5.9 \%(543 \pm 29 \text { vs. } 575 \pm 39 \mathrm{~W} ; \mathrm{d}=0.97[-11.79 \text { to }-0.01]) \\
\leftrightarrow \text { Total work }(\text { temperate }):-1.5 \%(67.44 \pm 14.39 \mathrm{vs.} 68.46 \pm 15.07 \mathrm{~kJ} ; \mathrm{d}=0.07[-0.91 \text { to } 0.78]) \\
\leftrightarrow \text { Total work }(\text { hot }):-5.6 \%(66.07 \pm 10.84 \text { vs. } 69.74 \pm 15.13 \mathrm{~kJ} ; \mathrm{d}=0.03[-1.12 \text { to } 0.58])\end{array}$ \\
\hline Thompson et al. [59] & $\begin{array}{l}16 \text { male recreational } \\
\text { team-sport players }\end{array}$ & $\begin{array}{l}2.5 \mathrm{~h} \text { prior to exercise and } \\
7 \mathrm{~d} \text { of } \mathrm{BR} \text { supplementation } \\
\left(\mathrm{NO}_{3}-12.8 \text { mmol per day }\right)\end{array}$ & $\begin{array}{c}2 \times 40 \text { min cycling sprints, } \\
15 \text { min rest } \\
10 \times 6 \mathrm{~s} \mathrm{sprints}, 100 \mathrm{~s} \text { rest } \\
\text { at } 35 \% \mathrm{VO}_{2 \max }+14 \mathrm{~s} \\
\text { passive rest } \\
5 \times 4 \mathrm{~s} \text { sprints, } 16 \mathrm{~s} \text { rest at } \\
35 \% \mathrm{VO}_{2 \max } \\
10 \times 6 \mathrm{~s} \mathrm{sprints}, 100 \mathrm{~s} \text { rest } \\
\text { at } 35 \% \mathrm{VO}_{2 \max }+14 \mathrm{~s} \\
\text { passive rest }\end{array}$ & $\uparrow$ Total work: $+3.5 \%(123 \pm 19$ vs. $119 \pm 17 \mathrm{~kJ} ; \mathrm{d}=0.23[-0.51$ to 0.94$])$ \\
\hline Wylie et al. [49] & $\begin{array}{l}10 \text { male recreational } \\
\text { team-sport players }\end{array}$ & $\begin{array}{l}2.5 \mathrm{~h} \text { prior to exercise and } 3 \\
\text { to } 5 \mathrm{~d} \text { of } \mathrm{BR} \\
\text { supplementation } \\
\left(\mathrm{NO}_{3}-8.2 \mathrm{mmol} \text { per day }\right)\end{array}$ & $\begin{array}{c}24 \times 6 \text { s cycling sprints } \\
24 \text { s rest }\end{array}$ & $\begin{array}{l}\leftrightarrow \text { Ppeak (mean): }+1.3 \%(792 \pm 159 \text { vs. } 782 \pm 154 \mathrm{~W} ; \mathrm{d}=0.07[-0.88 \text { to } 1.00]) \\
\uparrow \text { Pmean (sprints } 1-6):+7.3 \%(694 \pm 125 \text { vs. } 647 \pm 122 \mathrm{~W} ; \mathrm{d}=0.39[-0.58 \text { to } 1.31]) \\
\leftrightarrow \text { Pmean (sprints } 7-12):+3.9 \%(560 \pm 100 \text { vs. } 539 \pm 112 \mathrm{~W} ; \mathrm{d}=0.20[-0.75 \text { to } 1.13]) \\
\leftrightarrow \text { Pmean (sprints } 13-18):+5.3 \%(518 \pm 111 \text { vs. } 492 \pm 121 \mathrm{~W} ; \mathrm{d}=0.23[-0.73 \text { to } 1.16]) \\
\leftrightarrow \text { Pmean (sprints } 19-24):+4.8 \%(500 \pm 114 \text { vs. } 477 \pm 119 \mathrm{~W} ; \mathrm{d}=0.20[-0.75 \text { to } 1.13]) \\
\uparrow \text { Pmean (mean): }+5.4 \%(568 \pm 136 \text { vs. } 539 \pm 136 \mathrm{~W} ; \mathrm{d}=0.22[-0.74 \text { to } 1.15])\end{array}$ \\
\hline
\end{tabular}


Table 2. Cont.

Reference

Subjects

Supplementation

$2 \mathrm{~h}$ prior to exercise acute

$\mathrm{BR}$ ingestion

$\left(\mathrm{NO}_{3}-13 \mathrm{mmol}\right)$

Kent et al. [63]

12 male team sport players

7 healthy males

36 team sport players

Clifford et al. [64]

20 male team sport players

13 trained male

tennis players
$\mathrm{BR}$ ingestion

$\left(\mathrm{NO}_{3}{ }^{-} 6.45 \mathrm{mmol}\right)$

$2.5 \mathrm{~h}$ prior to exercise and $5 \mathrm{~d}$ of BR supplementation (12.8 $\mathrm{mmol} \mathrm{NO}_{3}{ }^{-}$per day)

$4 \mathrm{~d}$ of $\mathrm{BR}$ supplementation (2.31 $\mathrm{mmol} \mathrm{NO}_{3}{ }^{-}$per day) $3 \mathrm{~h}$ prior to exercise of acute $\mathrm{BR}$ ingestion
$3 \mathrm{~h}$ prior to exercise acute

Exercise Protocol

4 sets of $9 \times 4$ s cycling sprints with $16 \mathrm{~s}$ active $+6 \mathrm{~s}$ passive rest, interspersed with $3 \mathrm{~min}$

$$
\text { rest }(3000 \mathrm{~m}
$$

simulated altitude)

$5 \times 6$ s running sprints on treadmill, $30 \mathrm{~s}$ rest

$5 \times 20 \mathrm{~m}$ running sprints in running lanes, $30 \mathrm{~s}$ rest

$20 \times 30 \mathrm{~m}$ sprints, $30 \mathrm{~s}$ rest 5 tennis serves, $2 \times 10 \mathrm{~m}$ sprints, $2 \times$ agility test $(5-0-5), 1$ min rest

\section{Results}

$\leftrightarrow \mathrm{P}_{\text {peak }}$ (set 1$):-2.4 \%(1185 \pm 172$ vs. $1214 \pm 179 \mathrm{~W} ; \mathrm{d}=0.17$ [ -1.01 to 0.69$\left.]\right)$ $\leftrightarrow \mathrm{P}_{\text {peak }}($ set 1$):-2.1 \%(1157 \pm 178$ vs. $1181 \pm 163 \mathrm{~W} ; \mathrm{d}=0.15[-0.98$ to 0.71$])$ $\leftrightarrow \mathrm{P}_{\text {peak }}$ (set $):-1.0 \%(1159 \pm 186$ vs. $1165 \pm 160 \mathrm{~W} ; \mathrm{d}=0.04[-0.88$ to 0.81$])$ $\leftrightarrow \leftrightarrow \mathrm{P}_{\text {peak }}$ (set 3$):-0.5 \%(1152 \pm 194$ vs. $1164 \pm 139 \mathrm{~W} ; \mathrm{d}=0.07$ [ -0.92 to 0.78$\left.]\right)$ $\leftrightarrow \mathrm{P}_{\text {peak }}$ (set 4$):-1.0 \%(1152 \pm 194$ vs. $1164 \pm 139 \mathrm{~W} ; \mathrm{d}=0.07[-0.92$ to 0.78$])$
$\leftrightarrow \mathrm{P}_{\text {mean }}$ (set 1$):-2.7 \%(807 \pm 144$ vs. $829 \pm 144 \mathrm{~W} ; \mathrm{d}=0.16[-1.00$ to 0.70$])$ $\leftrightarrow \mathrm{P}_{\text {mean }}$ (set 2$):+3.1 \%(794 \pm 156$ vs. $770 \pm 142 \mathrm{~W} ; \mathrm{d}=0.17[-0.69$ to 1.00$])$ $\leftrightarrow \mathrm{P}_{\text {mean }}$ (set 3$):+2.1 \%(809 \pm 150$ vs. $792 \pm 131 \mathrm{~W} ; \mathrm{d}=0.15[-0.73$ to 0.96$])$ $\leftrightarrow P_{\text {mean }}$ (set 4$):-1.0 \%(779 \pm 156$ vs. $804 \pm 122 \mathrm{~W} ; \mathrm{d}=0.19$ [ -1.02 to 0.68$\left.]\right)$ $\leftrightarrow$ Total work (set 1$):-2.7 \%(29.0 \pm 5.18$ vs. $29.8 \pm 5.19 \mathrm{~J} ; \mathrm{d}=0.16$ [ -1.00 to 0.70$])$ $\leftrightarrow$ Total work (set 2$):-0.5 \%(28.5 \pm 5.61$ vs. $28.7 \pm 5.10 \mathrm{~J} ; \mathrm{d}=0.04$ [ -0.88 to 0.81$])$ $\leftrightarrow$ Total work (set 3$):+2.5 \%(29.1 \pm 5.38$ vs. $28.4 \pm 4.75 \mathrm{~J} \cdot \mathrm{d}=0.14$ [ -0.71 to 0.98$])$ $\leftrightarrow$ Total work (set 4$):+0.2 \%(28.9 \pm 5.62$ vs. $28.9 \pm 4.39 \mathrm{~J} ; \mathrm{d}=0.00$ [ -0.85 to 0.85$])$

$\leftrightarrow$ Work decrement (set 1$):-47.1 \%(11.9 \pm 6.9$ vs. $17.5 \pm 11.7 \% ; \mathrm{d}=0.48[-1.43$ to 0.30$])$ $\leftrightarrow$ Work decrement (set 1$):-47.1 \%(11.9 \pm 6.9$ vs. $17.5 \pm 11.7 \% ; \mathrm{d}=0.48[-1.43$ to 0.30$])$
$\leftrightarrow$ Work decrement (set 2$):-21.7 \%(12.9 \pm 9.1$ vs. $15.7 \pm 14.5 \% ; \mathrm{d}=0.47[-1.07$ to 0.63$])$ $\leftrightarrow$ Work decrement (set 3): $+4.5 \%$ (13.9 \pm 8.4 vs. $13.3 \pm 11 \%$; $d=0.09$ [ -0.79 to 0.91$])$ $\leftrightarrow$ Work decrement (set 4$):+2.5 \%$ (12.2 \pm 6.3 vs. $11.9 \pm 7.1 \% ; \mathrm{d}=0.05[-0.80$ to 0.89$])$

$\leftrightarrow \mathrm{P}_{\text {peak (mean) }}:+4.9 \%(4133.5 \pm 674.4$ vs. $3938.3 \pm 603.1 \mathrm{~W} ; \mathrm{d}=0.33$ [ -0.89 to 1.46$\left.]\right)$

Total time: $-1.2 \%(3.98 \pm 0.18$ vs. $4.03 \pm 0.19 \mathrm{~s} ; \mathrm{d}=0.27[-0.71$ to 0.20$])$

Time $(5 \mathrm{~m}):-2.3 \%(1.73 \pm 0.09$ vs. $1.77 \pm 0.09 \mathrm{~s} ; \mathrm{d}=0.45[-0.92$ to 0.04$])$

Time $(10 \mathrm{~m}):-1.6 \%(2.53 \pm 0.2$ vs. $2.57 \pm 0.12 \mathrm{~s} ; \mathrm{d}=0.25[-0.71$ to 0.23$])$

Time (5-10 m): $-1.2 \%(0.80 \pm 0.04$ vs. $0.81 \pm 0.04 \mathrm{~s} ; \mathrm{d}=0.25[-0.72$ to 0.22$])$

$\leftrightarrow$ Time $(10-20 \mathrm{~m}):-0.7 \%(1.45 \pm 0.07$ vs. $1.46 \pm 0.09 \mathrm{~s} ; \mathrm{d}=0.13$ [ -0.59 to 0.35$])$

$\leftrightarrow$ Best sprint time: $\mathrm{BR}-0.7 \%(4.38 \pm 0.17$ vs. $4.41 \pm 0.23 \mathrm{~s} ; \mathrm{d}=0.15$ [ -0.79 to 0.50$])$ and $\mathrm{PL}$

$+1.1 \%(4.53 \pm 0.15$ vs. $4.48 \pm 0.14 \mathrm{~s} ; \mathrm{d}=0.35[-0.31$ to 0.98$])$

$\leftrightarrow$ Serve speed: $-2.7 \%(160.6 \pm 10.4$ vs. $165.0 \pm 10.8 \mathrm{~km} / \mathrm{h} ; \mathrm{d}=0.15$ [ -1.22 to 0.42$])$

$\leftrightarrow$ Best sprint time $(10 \mathrm{~m}):+1.1 \%(1.86 \pm 0.07$ vs. $1.88 \pm 0.05 \mathrm{~s} ; \mathrm{d}=0.39$ [ -1.13 to 0.50$])$ $\leftrightarrow$ Best sprint time (5-0-5): $+2.0 \%(2.60 \pm 0.10$ vs. $2.64 \pm 0.10 \mathrm{~s} ; \mathrm{d}=0.69$ [ -1.21 to 0.43$])$

$\uparrow=$ significant increase; $\downarrow=$ significant decrease; $\leftrightarrow$ = no difference; $\mathrm{BR}=$ beetroot juice; $n$ =sample size; $\mathrm{NO}_{3}{ }^{-}=$nitrate; $\mathrm{PL}=$ placebo; $\mathrm{P}_{\max }=$ maximal power; $\mathrm{P}_{\text {mean }}=$ mean power $\mathrm{P}_{\text {peak }}=$ peak power; $\mathrm{RPM}_{\mathrm{opt}}=$ maximal optimal pedaling rate; $\mathrm{VO}_{2 \max }=$ maximal oxygen consumption; [ ] denotes confidence intervals. 
Table 3. Description of studies that have investigated the effects of $\mathrm{NO}_{3}{ }^{-}$supplementation on explosive resistance efforts in humans. There were 4 studies out of 10 that reported improved $P_{\text {peak }}$ in back squat [65], isokinetic knee flexion at 60\% /s [40], isokinetic knee extension at 6.28 rad/s [37], and during free-weight bench press [46] as well as $\mathrm{V}_{\text {mean }}$ of contraction during free-weight bench press exercise [46].

Reference
Rodríguez-Fernández et al. [65]
18 trained male athletes

$2.5 \mathrm{~h}$ prior to exercis acute $\mathrm{BR}$ ingestion $\left(\mathrm{NO}_{3}{ }^{-} 12.9 \mathrm{mmol}\right)$
$4 \times 8$ half squat in a flywheel device $(0.025$ 0.05 and $0.100 \mathrm{~kg} / \mathrm{m}^{2}$ ) with $3 \mathrm{~min}$ of rest
$2.5 \mathrm{~h}$ prior to exercise and $7 \mathrm{~d}$ of $\mathrm{BR}$

Tillin et al. [66]

Williams et al. [46] $\left(\mathrm{NO}_{3}-12.9 \mathrm{mmol}\right.$ per day) $\mathrm{BR}$ ingestion
17 male recreationally active athletes

11 resistance-trained male athletes
$2 \times$ concentric Smith-machine back squats and bench press a 2 min rest supplementation

$2 \mathrm{~h}$ prior to exercise of $\left(\mathrm{NO}_{3}{ }^{-} 6.4 \mathrm{mmol}\right)$

Exercise Protocol

$10 \times$ MIVC leg extensions 1 min rest

$2 \times 2$ at $70 \% 1 \mathrm{RM}$ 3 min rest Results

$\leftrightarrow$ Pmax (60\% 1RM) squat: $+1.8 \%$ (389 \pm 117 vs. $382 \pm 111 \mathrm{~W} ; \mathrm{d}=0.06$ [-0.79 to 0.91$])$ $\leftrightarrow \operatorname{Pmax}(70 \% 1 \mathrm{RM})$ squat: $-0.5 \%$ (393 \pm 116 vs. $395 \pm 107 \mathrm{~W} ; \mathrm{d}=0.02$ [ -0.83 to 0.83$])$ $\leftrightarrow P \operatorname{Pax}(80 \% 1 \mathrm{RM})$ squat: $-0.3 \%(377 \pm 108$ vs. $378 \pm 96 \mathrm{~W} ; \mathrm{d}=0.01$ [ -0.86 to 0.84$])$ $\leftrightarrow$ Pmax (60\% 1RM) bench press: $-1.0 \%$ (289 \pm 88 vs. $292 \pm 94 \mathrm{~W} ; \mathrm{d}=0.03$ [ -0.88 to 0.81$])$ $\leftrightarrow \operatorname{Pmax}(70 \% 1 \mathrm{RM})$ bench press: $+1.7 \%(242 \pm 81$ vs. $238 \pm 81 \mathrm{~W} ; \mathrm{d}=0.05$ [ -0.80 to 0.89$])$ $\leftrightarrow \operatorname{Pmax}(80 \% 1 \mathrm{RM})$ bench press: $-8.5 \%$ (176 \pm 66 vs. $191 \pm 55 \mathrm{~W} ; \mathrm{d}=0.26$ [ -1.09 to 0.61$])$ $\leftrightarrow \operatorname{Vmax}(60 \% 1 \mathrm{RM})$ squat: $+1.8 \%(0.70 \pm 0.09$ vs. $0.69 \pm 0.09 \mathrm{~m} / \mathrm{s} ; \mathrm{d}=0.14$ [ -0.74 to 0.95$])$ $\leftrightarrow \operatorname{Vmax}(70 \% 1 \mathrm{RM})$ squat: $+0.0 \%(0.61 \pm 0.08$ vs. $0.61 \pm 0.08 \mathrm{~m} / \mathrm{s} ; \mathrm{d}=0.08$ [ -0.85 to 0.85$])$ $\leftrightarrow \operatorname{Vmax}(80 \% 1 \mathrm{RM})$ squat: $+0.0 \%(0.51 \pm 0.09$ vs. $0.51 \pm 0.06 \mathrm{~m} / \mathrm{s} ; \mathrm{d}=0.01$ [ -0.85 to 0.85$])$ $\leftrightarrow \operatorname{Vmax}(60 \%$ 1RM) bench press: $+0.0 \%(0.61 \pm 0.08$ vs. $0.61 \pm 0.08 \mathrm{~m} / \mathrm{s} ; \mathrm{d}=0.04$ [ -0.85 to 0.85$])$ $\leftrightarrow \operatorname{Vmax}(60 \% 1 \mathrm{RM})$ bench press: $+0.0 \%(0.61 \pm 0.08$ vs. $0.61 \pm 0.08 \mathrm{~m} / \mathrm{s} ; \mathrm{d}=0.04[-0.85$ to 0.85$])$
$\leftrightarrow \operatorname{Vmax}(70 \% 1 \mathrm{RM})$ bench press: $+0.0 \%(0.43 \pm 0.06$ vs. $0.43 \pm 0.08 \mathrm{~m} / \mathrm{s} ; \mathrm{d}=0.03[-0.85$ to 0.85$])$

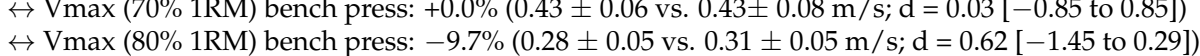
$\uparrow \mathrm{P}_{\text {peak CON }}\left(0.025 \mathrm{~kg} / \mathrm{m}^{2}\right):+16.4 \%(1251 \pm 249$ vs. $1075 \pm 205 \mathrm{~W} ; \mathrm{d}=0.79$ [0.05 to 1.46$\left.]\right)$ $\uparrow P_{\text {peak ECC }}\left(0.025 \mathrm{~kg} / \mathrm{m}^{2}\right):+18.9 \%(1195 \pm 265$ vs. $1005 \pm 176 \mathrm{~W} ; \mathrm{d}=0.87[0.12$ to 1.53$])$ $\uparrow \mathrm{P}_{\text {peak CON }}\left(0.050 \mathrm{~kg} / \mathrm{m}^{2}\right):+15.3 \%(1182 \pm 226$ vs. $1025 \pm 181 \mathrm{~W} ; \mathrm{d}=0.79$ [0.05 to 1.45$\left.]\right)$ $\uparrow P_{\text {peak ECC }}\left(0.050 \mathrm{~kg} / \mathrm{m}^{2}\right):+12.9 \%(1168 \pm 261$ vs. $1034 \pm 172 \mathrm{~W} ; \mathrm{d}=0.62$ [ -0.10 to 1.29$\left.]\right)$ $\uparrow \mathrm{P}_{\text {peak CON }}\left(0.075 \mathrm{~kg} / \mathrm{m}^{2}\right):+20.8 \%(1132 \pm 239$ vs. $937 \pm 158 \mathrm{~W} ; \mathrm{d}=0.99[0.23$ to 1.66$])$ $\uparrow P_{\text {peak ECC }}\left(0.075 \mathrm{~kg} / \mathrm{m}^{2}\right):+19.7 \%(1201 \pm 261$ vs. $1003 \pm 187 \mathrm{~W} ; \mathrm{d}=0.90$ [0.19 to 1.20$\left.]\right)$ $\uparrow \mathrm{P}_{\text {peak CON }}\left(0.100 \mathrm{~kg} / \mathrm{m}^{2}\right):+18.4 \%(1008 \pm 197$ vs. $851 \pm 161 \mathrm{~W} ; \mathrm{d}=0.90$ [0.14 to 1.56$\left.]\right)$ $\uparrow P_{\text {peak ECC }}\left(0.100 \mathrm{~kg} / \mathrm{m}^{2}\right):+12.0 \%(1070 \pm 230$ vs. $955 \pm 191 \mathrm{~W} ; \mathrm{d}=0.56$ [ -0.16 to 1.22$\left.]\right)$ $\uparrow P_{\text {mean CON }}\left(0.025 \mathrm{~kg} / \mathrm{m}^{2}\right):+16.4 \%(750 \pm 173$ vs. $644 \pm 153 \mathrm{~W} ; \mathrm{d}=0.67$ [ -0.06 to 1.33$\left.]\right)$ $\uparrow P_{\text {mean ECC }}\left(0.025 \mathrm{~kg} / \mathrm{m}^{2}\right):+19.6 \%(684 \pm 154$ vs. $572 \pm 131 \mathrm{~W} ; \mathrm{d}=0.81$ [0.06 to 1.47$\left.]\right)$ $\uparrow P_{\operatorname{mean} \text { ECC }}\left(0.025 \mathrm{~kg} / \mathrm{m}^{2}\right):+19.6 \%$ kg $\left./ \mathrm{m}^{2}\right):+18.6 \%(709 \pm 146$ vs. $598 \pm 140 \mathrm{~W} ; \mathrm{d}=0.80[0.06$ to 1.46$])$ $\uparrow \mathrm{P}_{\text {mean CON }}\left(0.050 \mathrm{~kg} / \mathrm{m}^{2}\right):+18.6 \%$ (709 $146 \mathrm{vs} .598 \pm 140 \mathrm{~W} ; \mathrm{d}=0.80[0.06$ to 1.46$\left.]\right)$ $\uparrow P_{\text {mean ECC }}\left(0.050 \mathrm{~kg} / \mathrm{m}^{2}\right):+17.8 \%(687 \pm 150$ vs. $583 \pm 162 \mathrm{~W} ; \mathrm{d}=0.69[-0.04$ to 1.35$])$ $\uparrow P_{\text {mean CON }}\left(0.075 \mathrm{~kg} / \mathrm{m}^{2}\right):+21.9 \%(672 \pm 157$ vs. $551 \pm 120 \mathrm{~W} ; \mathrm{d}=0.89[0.14$ to 1.56$])$ $\uparrow P_{\text {mean ECC }}\left(0.075 \mathrm{~kg} / \mathrm{m}^{2}\right):+22.2 \%(709 \pm 177$ vs. $580 \pm 145 \mathrm{~W} ; \mathrm{d}=0.82[0.08$ to 1.48$])$ $\uparrow P_{\text {mean CON }}\left(0.100 \mathrm{~kg} / \mathrm{m}^{2}\right):+21.7 \%(600 \pm 127$ vs. $493 \pm 120 \mathrm{~W} ; \mathrm{d}=0.89[0.14$ to 1.56$])$

$\uparrow \mathrm{P}_{\text {mean ECC }}\left(0.010 \mathrm{~kg} / \mathrm{m}^{2}\right):+13.9 \%(615 \pm 150$ vs. $540 \pm 139 \mathrm{~W} ; \mathrm{d}=0.53$ [ -0.18 to 1.20$\left.]\right)$

$\leftrightarrow$ Fmax: $+0.27 \%(741 \pm 136$ vs. $739 \pm 135 \mathrm{~N} ; \mathrm{d}=0.02[-0.68$ to 0.71$])$

$2 \times 2$ at $70 \% 1 \mathrm{RM}$
free-weight bench press, $\quad \begin{aligned} & \uparrow \text { Pmean: }+19.5 \%(607 \pm 112 \mathrm{vs.} 508 \pm 118 \mathrm{~W} ; \mathrm{d}=0.19[-0.10 \text { to } 1.76]) \\ & \text { 个 Vmean: }+6.5 \%(0.66 \pm 0.08 \text { vs. } 0.62 \pm 0.08 \mathrm{~m} / \mathrm{s} ; \mathrm{d}=0.52[-0.42 \text { to } 1.38])\end{aligned}$ 
Table 3. Cont.

\section{Reference}

$$
n=7)
$$

$2 \mathrm{~h}$ prior to exercise acute $\mathrm{BR}$ ingestion $\left(\mathrm{NO}_{3}{ }^{-} 11.2 \mathrm{mmol}\right)$

Supplementation

\section{Exercise Protocol}

2 sets $\times 5$ isometric knee extensions/flexions, $60^{\circ}$

flexion, $5 \mathrm{~s}$ rest,

$6 \mathrm{~d}$ of $\mathrm{KNO}_{3}$ $\left(\mathrm{NO}_{3}{ }^{-} 8\right.$ mmol per day $)$

$3 \mathrm{~h}$ prior to exercise and $6 \mathrm{~d}$ of BR supplementation per day)
p interspersed with

$2 \times 5$ isokinetic knee xtensions and flexions $60^{\circ} / \mathrm{s}$ and $180^{\circ} / \mathrm{s}$ 1 min rest

$5 \times$ CMJ, 1 min rest 5 isokinetic knee extensions and flexions at $60^{\circ} / \mathrm{s}, 120^{\circ} / \mathrm{s}, 180^{\circ} / \mathrm{s}$, and $300^{\circ} / \mathrm{s}$

$3 \times 4$ s MIVC leg extension with $30^{\circ}$ and $60^{\circ}$ of flexion, 1 min rest

3-4 isokinetic knee extensions at $0 \mathrm{rad} / \mathrm{s}$ $1.57 \mathrm{rad} / \mathrm{s}, 3.14 \mathrm{rad} / \mathrm{s}$

$4.17 \mathrm{rad} / \mathrm{s}$, and $6.28 \mathrm{rad} / \mathrm{s}, 2 \mathrm{~min}$ rest

$3 \mathrm{~h}$ prior to exercise acute $\mathrm{BR}$ ingestion
$\left(\mathrm{NO}_{3}-6.45 \mathrm{mmol}\right)$
Isokinetic knee extension and flexion at $60^{\circ} / \mathrm{s}$ and $240^{\circ} / \mathrm{s}$

\section{Results}

$\leftrightarrow$ Tpeak (isometric extension): $\mathrm{KNO}_{3}+10.2 \%(186 \pm 49$ vs. $169 \pm 37 \mathrm{~N} ; \mathrm{d}=0.42[-0.48$ to 1.23$])$ and PL $+6.1 \%(185 \pm 43$ vs. $174+28 \mathrm{~N} \cdot \mathrm{d}=0.31$ [ -0.56 to 1.14$])$

$\leftrightarrow$ Tpeak (isometric flexion): $\mathrm{KNO}_{3}+1.8 \%(119 \pm 27 \mathrm{vs.} 117 \pm 21 \mathrm{~N} ; \mathrm{d}=0.09$ [ -0.77 to 0.93$\left.]\right)$ and PL $+4.8 \%(126 \pm 20$ vs. $120 \pm 17 \mathrm{~N}$; d $=0.33$ [ -0.54 to 1.16$])$.

$\leftrightarrow$ Tpeak (extension at $\left.60^{\circ} / \mathrm{s}\right): \mathrm{KNO}_{3}-4.1 \%(168 \pm 50$ vs. $175 \pm 41 \mathrm{~N} ; \mathrm{d}=0.16[-1.00$ to 0.70$])$ and PL $-2.7 \%(179 \pm 44$ vs. $184 \pm 48.53 \mathrm{~N} ; \mathrm{d}=0.11$ [ -0.95 to 0.74$])$

$\leftrightarrow$ Tpeak (flexion at $\left.60^{\circ} / \mathrm{s}\right): \mathrm{KNO}_{3}-1.5 \%(102 \pm 26$ vs. $104 \pm 21 \mathrm{~N} ; \mathrm{d}=0.07$ [ -0.93 to 0.77$]$ ) and PL $-2.7 \%(104 \pm 25$ vs. $106 \pm 25 \mathrm{~N} ; \mathrm{d}=0.12$ [ -0.92 to 0.77$])$

$\leftrightarrow$ Tpeak (extension at $\left.180^{\circ} / \mathrm{s}\right): \mathrm{KNO}_{3}+6.5 \%(128 \pm 32$ vs. $120 \pm 36 \mathrm{~N} ; \mathrm{d}=0.24[-0.62$ to 1.08$])$ and $\mathrm{PL}+2.6 \%(123 \pm 35$ vs. $120 \pm 42 \mathrm{~N} ; \mathrm{d}=0.09$ [ -0.77 to 0.92$])$

$\leftrightarrow$ Tpeak (flexion at $\left.180^{\circ} / \mathrm{s}\right): \mathrm{KNO}_{3}+0.4 \%(80 \pm 16$ vs. $79 \pm 14 \mathrm{~N} ; \mathrm{d}=0.02$ [ -0.78 to 0.91$\left.]\right)$ and $\mathrm{PL}+0.8 \%(76 \pm 20$ vs. $76 \pm 28 \mathrm{~N} ; \mathrm{d}=0.02[-0.85$ to 0.85$])$

$\leftrightarrow \operatorname{Pmax}\left(\right.$ extension at $\left.60^{\circ} / \mathrm{s}\right):+0.9 \%(220 \pm 45$ vs. $218 \pm 40 \mathrm{~W} ; \mathrm{d}=0.05$ [ -0.73 to 0.82$\left.]\right)$

$\leftrightarrow \operatorname{Pmax}\left(\right.$ extension at $120^{\circ}$ /s): $+1.3 \%$ (392 \pm 74 vs. $387 \pm 62 \mathrm{~W} ; \mathrm{d}=0.08$ [ -0.71 to 0.85$\left.]\right)$

$\leftrightarrow P \operatorname{Pmax}\left(\right.$ extension at $\left.180^{\circ} / \mathrm{s}\right):+2.7 \%(500 \pm 86$ vs. $487 \pm 67 \mathrm{~W} ; \mathrm{d}=0.18$ [ $[-0.61$ to 0.94$])$

$\leftrightarrow \operatorname{Pmax}\left(\right.$ extension at $\left.300^{\circ} / \mathrm{s}\right):+1.8 \%(554 \pm 102$ vs. $544 \pm 81 \mathrm{~W} ; \mathrm{d}=0.11[-0.67$ to 0.88$]$ )

$\uparrow$ Pmax (flexion at $\left.60^{\circ} / \mathrm{s}\right):+2.0 \%$ (151 vs. $148 \mathrm{~W} ; \mathrm{d}=$ unknown)

$\leftrightarrow$ Pmax (flexion at $\left.120^{\circ} / \mathrm{s}\right):+1.3 \%$ (392 \pm 74 vs. $387 \pm 62 \mathrm{~W} ; \mathrm{d}=0.08$ [ -0.71 to 0.85$\left.]\right)$

$\leftrightarrow$ Pmax (flexion at $180^{\circ} / \mathrm{s}$ ): $+2.9 \%$ (391 \pm 57 vs. $380 \pm 58 \mathrm{~W} ; \mathrm{d}=0.20[-0.59$ to 0.96$\left.]\right)$

$\leftrightarrow P \operatorname{Pmax}\left(\right.$ flexion at $\left.300^{\circ} / \mathrm{s}\right):+1.6 \%(493+73$ vs. $485+81 \mathrm{~W} ; \mathrm{d}=0.11$ [ -0.68 to 0.88 ])

$\leftrightarrow \mathrm{Smax}$ (flexion of $\left.30^{\circ}\right):+2.0 \%(204+39$ vs. $200+37 \mathrm{Nm} ; \mathrm{d}=0.11[-0.68$ to 0.88$])$

S

$\leftrightarrow$ CMJ height: $-0.7 \%(39.3 \pm 6.3$ vs. $39.6 \pm 6.3 \mathrm{~cm} ; \mathrm{d}=0.05[-0.82$ to 0.73$])$

$\leftrightarrow$ GRFmax: $-0.5 \%$ (3.04 vs. $3.06 \mathrm{~N}$; d = unknown)

$\leftrightarrow \mathrm{P}_{\text {peak }}($ extension at $1.57 \mathrm{rad} / \mathrm{s}):-2.1 \%(3.31 \pm 0.16$ vs. $3.38 \pm 0.21 \mathrm{~W} / ; \mathrm{d}=0.39$ [ -1.22 to 0.49$\left.]\right)$ $\leftrightarrow \mathrm{P}_{\text {peak }}$ (extension at $\left.3.14 \mathrm{rad} / \mathrm{s}\right):-1.9 \%(5.38 \pm 0.32$ vs. $5.48 \pm 0.38 \mathrm{~W} / \mathrm{kg} ; \mathrm{d}=0.30[-1.13$ to 0.58$])$ $\leftrightarrow \mathrm{P}_{\text {peak }}($ extension at $4.17 \mathrm{rad} / \mathrm{s}):+0.0 \%(6.67 \pm 0.46$ vs. $6.67 \pm 0.50 \mathrm{~W} / \mathrm{kg} ; \mathrm{d}=0.00[-0.85$ to 0.85$])$ $\uparrow \mathrm{P}_{\text {peak }}$ (extension at $\left.6.28 \mathrm{rad} / \mathrm{s}\right):+4.1 \%(7.64 \pm 0.52 \mathrm{vs} .7 .34 \pm 0.54 \mathrm{~W} / \mathrm{kg} ; \mathrm{d}=0.59$ [ -0.32 to 1.41$\left.]\right)$ $\leftrightarrow \mathrm{T}_{\text {peak }}$ (extension at $\left.1.57 \mathrm{rad} / \mathrm{s}\right):-1.9 \%(2.11 \pm 0.10$ vs. $2.15 \pm 0.11 \mathrm{Nm} / \mathrm{kg} ; \mathrm{d}=0.40[-1.22$ to 0.49$])$ $\leftrightarrow \mathrm{T}_{\text {peak }}$ (extension at $\left.3.14 \mathrm{rad} / \mathrm{s}\right):-1.8 \%(1.71 \pm 0.10 \mathrm{vs} .1 .74 \pm 0.12 \mathrm{Nm} / \mathrm{kg} ; \mathrm{d}=0.28$ [-1.11 to 0.59$\left.]\right)$ 作 $\leftrightarrow \mathrm{T}_{\text {peak }}$ (extension at $\left.6.28 \mathrm{rad} / \mathrm{s}\right):+4.3 \%(1.22 \pm 0.08$ vs. $1.17 \pm 0.08 \mathrm{Nm} / \mathrm{kg} ; \mathrm{d}=0.65[-0.26$ to 1.47$])$ $\leftrightarrow \mathrm{T}_{\max }(0 \mathrm{rad} / \mathrm{s}):-1.5 \%(2.6 \pm 0.13$ vs. $2.64 \pm 0.13 \mathrm{Nm} / \mathrm{kg} ; \mathrm{d}=0.32$ [ -1.15 to 0.55$\left.]\right)$

$\leftrightarrow \mathrm{T}_{\text {peak }}$ (extension at $\left.60^{\circ} / \mathrm{s}\right):-2.6 \%(200.2 \pm 25.8$ vs. $207.4 \pm 37.5 \mathrm{Nm} ; \mathrm{d}=0.24$ [ -1.38 to 0.96$]$ $\leftrightarrow \mathrm{T}_{\text {peak }}$ (extension at $\left.240^{\circ} / \mathrm{s}\right):-5.9 \%(124.1 \pm 9.2$ vs. $131.4 \pm 17.1 \mathrm{Nm} ; \mathrm{d}=0.57$ [ -1.68 to 0.69$\left.]\right)$ $\leftrightarrow \mathrm{T}_{\text {peak }}$ (flexion at $\left.60^{\circ} / \mathrm{s}\right):-7.4 \%(103.3 \pm 27.7$ vs. $110.9 \pm 29.9 \mathrm{Nm} ; \mathrm{d}=0.28$ [ -1.42 to 0.92$\left.]\right)$ $\leftrightarrow \mathrm{T}_{\text {peak }}$ (flexion at $\left.240^{\circ} / \mathrm{s}\right):-16.1 \%(59.8 \pm 29.5$ vs. $69.4 \pm 21.5 \mathrm{Nm} ; \mathrm{d}=0.40$ [-1.52 to 0.83$]$ 
Table 3. Cont.

\begin{tabular}{|c|c|c|c|c|}
\hline Reference & Subjects & Supplementation & Exercise Protocol & Results \\
\hline López-Samanes et al. [57] & $\begin{array}{l}13 \text { trained male } \\
\text { tennis players }\end{array}$ & $\begin{array}{l}3 \mathrm{~h} \text { prior to exercise } \\
\text { acute BR ingestion } \\
\left(\mathrm{NO}_{3}^{-}-6.4 \mathrm{mmol}\right)\end{array}$ & $\begin{array}{l}2 \text { MIVC handgrip } \\
3 \text { CMJ with } 45 \text { s of rest }\end{array}$ & $\begin{array}{l}\leftrightarrow \mathrm{S}_{\max } \text { (handgrip): }+3.9 \%(47.8 \pm 9.3 \text { vs. } 46.0 \pm 7.9 \mathrm{~kg} ; \mathrm{d}=0.26[-0.61 \text { to } 1.01]) \\
\leftrightarrow \text { CMJ height: }+2.5 \%(33.0 \pm 4.9 \text { vs. } 32.2 \pm 5.1 \mathrm{~cm} ; \mathrm{d}=0.143[-0.66 \text { to } 0.97])\end{array}$ \\
\hline Haider et al. [68] & 19 healthy males & $\begin{array}{c}2.5 \mathrm{~h} \text { prior to exercise } \\
\text { and } 7 \mathrm{~d} \text { of } \mathrm{BR} \\
\text { supplementation } \\
\left(\mathrm{NO}_{3}-\sim 9.7 \mathrm{mmol}\right. \\
\text { per day) }\end{array}$ & $\begin{array}{l}\quad 4 \times 3 \mathrm{~s} \text { MIVC leg } \\
\text { extension with } 110^{\circ} \text { of } \\
\text { flexion with } \geq 30 \mathrm{~s} \text { rest } \\
15 \times 1 \mathrm{~s} \text { isometric knee } \\
\text { extensions with } \geq 15 \mathrm{~s} \text { rest }\end{array}$ & $\leftrightarrow \mathrm{F}_{\max }$ : (value not specified; $\mathrm{d}=$ unknown) \\
\hline
\end{tabular}

$\uparrow=$ significant increase; $\downarrow=$ significant decrease; $\leftrightarrow=$ no difference; $\mathrm{BR}=$ beetroot juice; $\mathrm{CMJ}=$ countermovement jumps; $\mathrm{CON}=$ concentric; $\mathrm{ECC}=$ eccentric; $\mathrm{F}_{\text {max }}=$ maximal force; $\mathrm{GRF}_{\max }=$ peak ground reaction force; $\mathrm{MIVC}=$ maximal isometric voluntary contraction; $n=$ sample size; $\mathrm{NO}_{3}{ }^{-}=$nitrate; $\mathrm{PL}=$ placebo; $\mathrm{P}_{\max }=$ maximal power; $\mathrm{P}_{\text {mean }}=$ mean power; $\mathrm{P}_{\text {peak }}=$ peak power; $\mathrm{RM}=$ repetition maximum; $\mathrm{SJ}=$ squat height; $\mathrm{S}_{\max }=$ maximal strength; $\mathrm{T}_{\text {peak }}=$ peak torque, $\mathrm{V}_{\text {max }}=$ maximal velocity; $\mathrm{V}_{\text {mean }}=$ mean velocity; [ ] denotes confidence intervals.

Table 4. Quality assessment of studies.

\begin{tabular}{|c|c|c|c|c|c|c|c|c|c|c|c|c|c|}
\hline Reference & Item 1 & Item 2 & Item 3 & Item 4 & Item 5 & Item 6 & Item 7 & Item 8 & Item 9 & Item 10 & Item 11 & Item 12 & Score \\
\hline Buck et al. [61] & Yes & Yes & Yes & Yes & Yes & Yes & Yes & Yes & Yes & Yes & Yes & No & $10 / 11$ \\
\hline Rimer et al. [44] & Yes & Yes & Yes & Yes & Yes & Yes & Yes & Yes & Yes & Yes & Yes & No & $10 / 11$ \\
\hline Smith et al. [62] & Yes & Yes & Yes & Yes & Yes & Yes & Yes & Yes & Yes & Yes & Yes & No & $10 / 11$ \\
\hline Thompson et al. [60] & Yes & Yes & Yes & Yes & Yes & Yes & No & Yes & Yes & Yes & Yes & No & $9 / 11$ \\
\hline Wylie et al. [49] & Yes & Yes & Yes & Yes & Yes & Yes & No & Yes & Yes & Yes & Yes & No & $9 / 11$ \\
\hline Kent et al. [63] & No & No & Yes & No & Yes & Yes & No & Yes & Yes & Yes & Yes & No & $7 / 11$ \\
\hline Kokkinoplitis et al. [56] & Yes & Yes & Yes & Yes & Yes & Yes & No & Yes & Yes & Yes & Yes & No & $9 / 11$ \\
\hline Thompson et al. [59] & Yes & Yes & Yes & Yes & Yes & Yes & No & Yes & Yes & Yes & Yes & No & $9 / 11$ \\
\hline Clifford et al. [64] & Yes & Yes & Yes & Yes & Yes & Yes & Yes & Yes & Yes & Yes & Yes & No & $10 / 11$ \\
\hline López-Samanes et al. [57] & Yes & Yes & Yes & Yes & Yes & Yes & No & Yes & Yes & Yes & Yes & No & $9 / 11$ \\
\hline Ranchal-Sánchez et al. [47] & Yes & Yes & Yes & Yes & Yes & Yes & Yes & Yes & Yes & Yes & Yes & No & $10 / 11$ \\
\hline Rodríguez-Fernández et al. [65] & Yes & Yes & Yes & Yes & Yes & Yes & No & Yes & Yes & Yes & Yes & No & $9 / 11$ \\
\hline Tillin et al. [66] & Yes & Yes & Yes & Yes & Yes & Yes & Yes & Yes & Yes & Yes & Yes & No & $10 / 11$ \\
\hline Williams et al. [46] & Yes & Yes & Yes & Yes & Yes & Yes & Yes & Yes & Yes & Yes & Yes & No & $10 / 11$ \\
\hline Kramer et al. [67] & Yes & Yes & Yes & Yes & Yes & Yes & Yes & Yes & Yes & Yes & Yes & No & $10 / 11$ \\
\hline Jonvik et al. [40] & Yes & Yes & Yes & Yes & Yes & Yes & Yes & Yes & Yes & Yes & Yes & No & $10 / 11$ \\
\hline Coggan et al. [37] & Yes & Yes & Yes & Yes & Yes & Yes & No & Yes & Yes & Yes & Yes & No & $9 / 11$ \\
\hline Haider et al. [68] & Yes & Yes & Yes & Yes & Yes & Yes & Yes & Yes & Yes & Yes & Yes & No & $10 / 11$ \\
\hline
\end{tabular}


Resistance-based exercise protocols included half squats using a flywheel device [65], bench press using a Smith machine [47], back squat and bench press using free weights [46], isometric $[40,57,66-68]$ or isokinetic $[37,40,56,67]$ knee flexions and extensions, isometric handgrip contractions [57], and countermovement jumps (CMJ) [40,57]. The recovery periods between efforts ranged from $15 \mathrm{~s}[68]$ to $3 \mathrm{~min}[46,65]$.

With the exception of Kramer et al. [67], in which potassium $\mathrm{NO}_{3}{ }^{-}$salts $\left(\mathrm{KNO}_{3}\right)$ were administered, beetroot juice was the source of $\mathrm{NO}_{3}{ }^{-}$for all remaining studies. All placebo interventions used in the reviewed studies had negligible $\mathrm{NO}_{3}{ }^{-}$content. In most cases $[37,40,44,49,59,61-63,65,66]$ concentrated beetroot juice was administered for the experimental and placebo condition, where the placebo consisted of $\mathrm{NO}_{3}{ }^{-}$-depleted concentrated beetroot juice. In other studies, the placebo was administered as blackcurrant juice $[46,47,56,68]$, potassium chloride tablets [67], beetroot juice diluted in water [57], and juices with a similar caloric and macronutrient content (but different in antioxidants, texture, and taste) in the control group [64].

Both acute [37,44,46,47,57,62,63,65] and chronic [40,49,59-61,64,66-68] supplementation protocols were employed in the reviewed studies. $\mathrm{NO}_{3}{ }^{-}$dosing ranged from $6.2 \mathrm{mmol}$ [62] to $12.9 \mathrm{mmol}[62,65]$ of $\mathrm{NO}_{3}{ }^{-}$for acute protocols, and from $6 \mathrm{mmol}$ [61] to $15.8 \mathrm{mmol}$ [40] for chronic protocols. $\mathrm{NO}_{3}{ }^{-}$supplementation was administered $2-3 \mathrm{~h}$ before testing for the acute protocols, whereas multi-day $\mathrm{NO}_{3}{ }^{-}$supplementation occurred in a range of short-term (3 days) $[59,64]$ to chronic (7 days) $[59,66,68]$ strategies before the onset of testing.

\section{Discussion}

This is the first systematic review to have examined whether dietary $\mathrm{NO}_{3}{ }^{-}$supplementation can enhance exercise performance during explosive efforts lasting for $\leq 6 \mathrm{~s}$ in various modes of exercise. The main finding was that dietary $\mathrm{NO}_{3}{ }^{-}$ingestion has the potential to increase power output, sprint time, and total work in sprint-type exercise, as well as power and velocity of contraction in resistance-type exercise, but that these effects are likely impacted by factors affecting NO bioavailability, exercise modality, and participant characteristics.

\subsection{The Effects of Dietary Nitrate on Explosive Sprinting Exercise Performance}

Out of the 10 studies that investigated explosive efforts during sprinting exercise, there were 5 studies that administered short-term ( 3 days) and chronic ( $\geq 3$ days) dietary $\mathrm{NO}_{3}{ }^{-}$ supplementation $[49,59-61,64]$, while the other 5 studies provided acute $\mathrm{NO}_{3}{ }^{-}$ingestion ( 2-3 h prior to exercise) $[44,56,57,62,63]$. There were four studies that observed significant performance improvements to sprints in cycling [44,49,59] and running [60]. Specifically, $\mathrm{NO}_{3}{ }^{-}$improved cycling maximal power $\left(\mathrm{P}_{\max }\right)$ [44], optimal repetitions per minute $\left(\mathrm{RPM}_{\mathrm{opt}}\right)$ [44], $\mathrm{P}_{\text {mean }}$ [49], and total work performed [59]. Interestingly, three of these studies provided $\mathrm{NO}_{3}{ }^{-}$chronically, ranging from 3 [49,60] to 7 days [59], whilst Rimer et al. [44] administered $\mathrm{NO}_{3}{ }^{-}$acutely $\sim 2-3 \mathrm{~h}$ prior to exercise. However, there were two studies that administered chronic $\mathrm{NO}_{3}{ }^{-}$supplementation that did not report improvements to sprint times [61,64]. Clifford et al. [64] provided 3 days of $\mathrm{NO}_{3}{ }^{-}$supplementation to healthy males, but importantly, the $\mathrm{NO}_{3}{ }^{-}$dose reported (143 $\mathrm{mg}$ or $\sim 2.3 \mathrm{mmol}$ of $\mathrm{NO}_{3}{ }^{-}$) is markedly lower than the findings of a dose-response study, where $8.4 \mathrm{mmol}$ of $\mathrm{NO}_{3}{ }^{-}$was evidenced to be the minimum amount required to induce physiological effects [11].

There is evidence that the magnitude of elevation in plasma $\left[\mathrm{NO}_{2}{ }^{-}\right]$(and thus $\mathrm{NO}$ bioavailability) is associated with improvements to muscular work and capacity $[25,39,69,70]$, but it is acknowledged that other factors may have a role in a lack of effect. In support of this notion, with 6 days of $\mathrm{NO}_{3}{ }^{-}$supplementation, Buck et al. [61] did not observe improvements to sprint time in female amateur team sport players, but it could be possible that the experiment was underpowered given that the power calculation was based on an experiment with a rowing exercise protocol [71] when the study employed running sprints. For example, running sprints were improved in Thompson et al. [60] where 36 participants were recruited 
compared to the 13 participants recruited in Buck et al. [61]. Furthermore, it could be possible that the inclusion of female participants (wherein nine females were not on hormonal contraceptives) had a role in the discrepancy. Importantly, chronic $\mathrm{NO}_{3}{ }^{-}$ingestion is likely not the sole factor in determining the efficacy of $\mathrm{NO}_{3}{ }^{-}$in performance during explosive sprinting exercise. However, given that most studies that did not observe effects employed acute $\mathrm{NO}_{3}{ }^{-}$dosing regimens, these data could highlight supplementation duration as a methodological consideration for $\mathrm{NO}_{3}{ }^{-}$-induced effects on explosive sprinting performance. Together, these observations could suggest that explosive sprint efforts in cycling and/or running could require consecutive multi-day $\mathrm{NO}_{3}{ }^{-}$loading to induce performance effects. Clearly, further research is warranted to understand the optimal dosing guidelines for $\mathrm{NO}_{3}{ }^{-}$ supplementation and whether there is an influence on explosive sprinting efforts.

\subsection{The Effects of Dietary Nitrate on Explosive Resistance Exercise Performance}

Out of the 10 studies that employed explosive efforts during resistance-type exercise, 6 of the studies administered $\mathrm{NO}_{3}{ }^{-}$acutely (2 to $3 \mathrm{~h}$ prior to exercise) while 4 of the studies administered chronic $\mathrm{NO}_{3}{ }^{-}$supplementation over 6 days [40] or 7 days [66,68]. There were four studies that reported performance improvements to peak power $\left(\mathrm{P}_{\text {peak }}\right)$ in squat exercise using a fly wheel device (+12-22\%) [65] isokinetic knee flexion at $60^{\circ} / \mathrm{s}$ $(+2 \%)$ [40], isokinetic knee extension at $6.28 \mathrm{rad} / \mathrm{s}(+4 \%)$ [37], and during free-weight bench press $(+19 \%)$ [46]. In addition, the mean velocity $\left(\mathrm{V}_{\text {mean }}\right)$ of contraction was enhanced $(+7 \%)$ during free-weight bench press exercise [46]. Interestingly, three of the four studies that observed performance enhancements in resistance-type exercise employed acute $\mathrm{NO}_{3}{ }^{-}$supplementation $[38,46,65]$. These data are in contrast to the supplementation regimens (short-term and chronic $\mathrm{NO}_{3}{ }^{-}$supplementation) reported in studies that observed improved explosive efforts during sprinting exercise following $\mathrm{NO}_{3}{ }^{-}$supplementation. Moreover, despite two of the studies providing the same $\mathrm{NO}_{3}{ }^{-}$dose $(1 \times 70 \mathrm{~mL}$ of $6.4 \mathrm{mmol}$ of $\mathrm{NO}_{3}{ }^{-}$) for bench press exercise, Ranchal-Sanchez et al. [47] did not observe any improvements to power or velocity of contraction at $60 \% 1 \mathrm{RM}, 70 \% 1 \mathrm{RM}$, or $80 \% 1 \mathrm{RM}$, compared to the $\sim 19 \%$ and $\sim 7 \%$ improvement to power and velocity of bench press at $70 \% 1 \mathrm{RM}$ [46] following $\mathrm{NO}_{3}{ }^{-}$ingestion. In knee extension and flexion exercise protocols, there is conflicting data given that acute [56] and chronic $\mathrm{NO}_{3}{ }^{-}$supplementation (6-7 days) did not impact peak torque $\left(\mathrm{T}_{\text {peak }}\right)$ [67] or maximal force $\left(\mathrm{F}_{\max }\right)$ [66,68], but flexion [40] and extension [37] at specific angular velocities exhibited a $\sim 2-4 \%$ improvement to $P_{\text {peak }}$. Lastly, handgrip strength [57] and countermovement jump (CMJ) performance [40,57] were not influenced by $\mathrm{NO}_{3}{ }^{-}$. These discrepancies are difficult to explain given the limited evidence available surrounding the effects of $\mathrm{NO}_{3}{ }^{-}$ingestion on resistance exercise, and there are considerable differences in study designs between the reviewed studies. However, potential factors influencing the efficacy $\mathrm{NO}_{3}{ }^{-}$on sprint- and resistance-type explosive exercise are discussed below.

\subsection{Potential Factors Influencing Nitrate-Induced Improvements in Explosive Exercise}

It is unclear as to why improved explosive efforts in sprint-based exercise occurred in more studies with short-term and chronic $\mathrm{NO}_{3}{ }^{-}$supplementation dosing regimens, whereas improved explosive efforts in resistance-based exercise occurred in more studies with acute $\mathrm{NO}_{3}{ }^{-}$dosing strategies. However, these data highlight that exercise modality is likely a factor that influences the efficacy of dietary $\mathrm{NO}_{3}{ }^{-}$supplementation and emphasizes the need for further research in this area. In the studies that incorporated weightlifting, the participants were resistance-trained compared to the team sport players of the studies with sprinting exercise; thus, it could be possible that differences in the training history of participants impacted motor unit recruitment patterns [48] and muscle fiber type composition $[72,73]$. This is in line with recent speculation that $\mathrm{NO}_{3}{ }^{-}$supplementation has a greater efficacy during the initial phases of contraction during force production [36,41-43] and thus the speed of acceleration of movements, which could be training-dependent. Other factors such as biomechanics, motor skills, delivery of instructions, and equipment might 
contribute to influence the efficacy of $\mathrm{NO}_{3}{ }^{-}$on performance between explosive sprint and resistance exercise [74]. Furthermore, differences in the recruitment of upper and lower body musculature during exercise [71] may be a factor to explore given that fiber type seems to impact the efficacy of $\mathrm{NO}_{3}{ }^{-}$on physiology $[28,29]$.

\subsubsection{Nitric Oxide Bioavailability: Supplementation Strategies}

The efficacy of dietary $\mathrm{NO}_{3}{ }^{-}$supplementation is thought to be linked to the magnitude of elevation in NO bioavailability, which could partly account for the discrepancies in the literature and reviewed studies above. NO bioavailability is often measured through the surrogate marker of plasma $\left[\mathrm{NO}_{2}{ }^{-}\right]$, such that a greater increase in plasma $\left[\mathrm{NO}_{2}{ }^{-}\right]$has been associated with improved muscular work or capacity $[39,69,70]$. However, there are several possible factors that influence $\mathrm{NO}$ bioavailability such as dosing regimen (i.e., timing, acute vs. chronic and total $\mathrm{NO}_{3}{ }^{-}$provided), where it is now widely accepted that a dose of $>6 \mathrm{mmol}$ of $\mathrm{NO}_{3}{ }^{-}$is required [11] and that there may be additional performance benefits with larger doses [25,69]. In addition, the vehicle of administration (i.e., juices vs. salts) could be influential on the efficacy of $\mathrm{NO}_{3}{ }^{-}$given that $\mathrm{NO}_{3}{ }^{-}$-rich beetroot juice contains bioactive phytochemicals such as flavonoids, betalains, and ascorbic acid [75]. Although speculative, the presence of these antioxidant constituents could further elevate $\mathrm{NO}$ given that polyphenols facilitate the reduction of $\mathrm{NO}_{2}{ }^{-}$to $\mathrm{NO}$ [76] or induce synergistic effects [15]. Flueck et al. [77] reported that the administration of $\mathrm{NO}_{3}{ }^{-}$-rich beetroot juice improved oxygen consumption during exercise compared to $\mathrm{NO}_{3}{ }^{-}$salts, supporting the notion that juices may be more effective, but further work is required. The training status of participants [78] may be an influential factor given that redox status impacts NO bioavailability [79] and that baseline plasma $\left[\mathrm{NO}_{2}{ }^{-}\right]$could be higher in elite athletes due to differences in habitual diet in highly trained populations [80]. Lastly, it is unknown whether NO bioavailability (or other physiological mechanisms) is influenced when dietary $\mathrm{NO}_{3}{ }^{-}$is co-ingested with other ergogenic aids. In the limited available data thus far, no synergistic effects on exercise performance have been reported when beetroot juice was co-ingested with caffeine [81-83] or sodium bicarbonate [84], but further research is warranted to understand whether combining multiple ergogenic aids or other nutritional strategies with dietary $\mathrm{NO}_{3}{ }^{-}$could impact the effects of dietary $\mathrm{NO}_{3}{ }^{-}$on performance in various exercise modalities and participant populations.

\subsubsection{Nitric Oxide Bioavailability: Skeletal Muscle Modulations and Storage}

The effects of multi-day $\mathrm{NO}_{3}{ }^{-}$ingestion may be important for $\mathrm{NO}$ bioavailability by providing a time window for structural and functional changes to proteins responsible for excitation-contraction coupling [35]. For example, acute $\mathrm{NO}_{3}{ }^{-}$ingestion could induce signaling effects to alter myofibrillar protein function [15], but $\mathrm{NO}_{3}{ }^{-}$loading over several days could potentially provide further augmentations through increased $\mathrm{NO}_{3}{ }^{-}$ content stored in skeletal muscle [85]. In addition, multi-day $\mathrm{NO}_{3}{ }^{-}$loading could alter expression and/or function of proteins regulating calcium release from the sarcoplasmic reticulum $[28,29]$. However, to date, chronic $\mathrm{NO}_{3}{ }^{-}$supplementation has not been observed to change calcium handing proteins in humans [30], and most data on skeletal muscle $\mathrm{NO}_{3}{ }^{-}$and $\mathrm{NO}_{2}{ }^{-}$are from rodent studies [86-88]. The ability for skeletal muscle to serve as a $\mathrm{NO}_{3}{ }^{-}$storage site is an attractive notion given that the $\mathrm{NO}_{3}{ }^{-}{ }^{-} \mathrm{NO}_{2}{ }^{-}{ }^{-} \mathrm{NO}$ pathway would enable manipulation of stored muscle $\mathrm{NO}_{3}{ }^{-}$through the ingestion of dietary $\mathrm{NO}_{3}{ }^{-}$and, thus, potentially improve local utilization of $\mathrm{NO}$ from $\mathrm{NO}_{3}{ }^{-}$reserves. In humans, recent advances show that $\mathrm{NO}_{3}{ }^{-}$content is significantly greater in skeletal muscle compared to plasma, and that ingestion of $\sim 13 \mathrm{mmol}$ of $\mathrm{NO}_{3}{ }^{-}$increased muscle $\mathrm{NO}_{3}{ }^{-}$by five-fold [89]. Moreover, the skeletal muscle $\mathrm{NO}_{3}{ }^{-}$concentration declined by 39\% following high-intensity exercise to exhaustion, highlighting the potential role of muscle in localized NO production during exercise [89]. Further work is required to understand the mechanistic bases of $\mathrm{NO}_{3}{ }^{-}$in muscle function, as well as the role of skeletal muscle $\mathrm{NO}_{3}{ }^{-}$ 
and whether altering muscle $\mathrm{NO}_{3}{ }^{-}$content is associated with improvements to muscle function and exercise performance.

\subsubsection{Nitric Oxide Bioavailability: Oral Microbiome}

The role of the oral microbiome in enhancing $\mathrm{NO}$ bioavailability is not well understood but may have important implications for the $\mathrm{NO}_{3}{ }^{-}{ }^{-} \mathrm{NO}_{2}{ }^{-}-\mathrm{NO}$ pathway and thus $\mathrm{NO}$ homeostasis [90]. For example, the use of oral hygiene products (e.g., mouthwash [91,92]) or elevation in thiocyanate, a compound competing $\mathrm{NO}_{3}{ }^{-}$transporters (e.g., smoking [93]), has been evidenced to disrupt $\mathrm{NO}_{3}{ }^{-}$metabolism, consequently attenuating the characteristic elevation in plasma $\left[\mathrm{NO}_{2}{ }^{-}\right]$following $\mathrm{NO}_{3}{ }^{-}$ingestion and abolishing potential positive $\mathrm{NO}_{3}{ }^{-}$-induced effects, such as the lowering of blood pressure [93]. Recent advances suggest that $\mathrm{NO}_{3}{ }^{-}$ingestion could favorably alter the microbial composition such that $\mathrm{NO}_{3}{ }^{-}$ reducing taxa are increased [94], and thus it could be reasoned that, consequently, elevations in $\mathrm{NO}_{2}{ }^{-}$could be more pronounced following multi-day ingestion of $\mathrm{NO}_{3}{ }^{-}$as microbial composition is modulated. However, the exact impact of $\mathrm{NO}_{3}{ }^{-}$dose and duration, as well as the oral microbiota species on influencing $\mathrm{NO}$ bioavailability and muscle physiology, is still elusive and requires further research.

\subsubsection{Nitric Oxide Bioavailability: Sex Differences}

In the reviewed studies, 20 out of 267 participants were female, which is equivalent to only $\sim 7 \%$ of the total number of participants as females. It is extremely unfortunate that females have been underappreciated in sports and exercise medicine research for so long [95], and thus it is unsurprising that the impact of sex differences on the efficacy of dietary $\mathrm{NO}_{3}{ }^{-}$remains unexplored. However, the scientific community is progressively becoming cognizant of potential sex differences in $\mathrm{NO}_{3}{ }^{-}$research, and open questions for investigation include examining sex-based physiological [96] and hormonal [97] influences on the mechanisms and performance effects of $\mathrm{NO}_{3}{ }^{-}$. To date, only eight studies have exclusively included females in dietary $\mathrm{NO}_{3}{ }^{-}$research, and there are inconsistencies between studies regarding the control for the menstrual cycle and hormonal contraceptives [see review: 96]. These controls are further complicated as there is currently no consensus on whether oral contraceptive use and/or the menstrual cycle affect strength and power performance, thus potentially under-powering studies that assess explosive power in female-only cohorts. One important sex difference was reported by Kapil et al. [98] such that the oral microbiome in females has an increased capacity to reduce $\mathrm{NO}_{3}{ }^{-}$into $\mathrm{NO}_{2}{ }^{-}$. However, whether this physiological difference has an influence on the bioavailability and bioactivity of $\mathrm{NO}$ and its subsequent effects on $\mathrm{NO}_{3}{ }^{-}$-induced mechanisms and performance have yet to be elucidated, making this a ripe area for research. Lastly, although hormonal controls may be barriers to include females, scientists are encouraged to examine if their laboratories are capable of incorporating as many methodological considerations for testing females if possible [97].

\subsubsection{Methodology}

In the selection of an exercise protocol during the study design phase, the tests have to be feasible for acquiring relevant physiological mechanistic data and to sufficiently induce the correct metabolic demand for the sport discipline of interest. It is important to note that the reliability and validity of exercise tests (test-retest repeatability over multiple trials) is crucial for the detection of 'real' changes between an intervention and control group, especially for small performance changes, as well as to minimize inaccurate interpretations of results. However, there is a paucity of repeatability data reported within dietary $\mathrm{NO}_{3}{ }^{-}$ research [59] and in the literature surrounding various high-intensity protocols [99-103]. Furthermore, given the obvious translation of high-intensity exercise protocols to numerous sports, there are infinite work-to-rest combinations for designing high-intensity exercise protocols, requiring many validation studies to account for each protocol. Thus far, the repeatability of data may be improved by the inclusion of familiarization trials [99], as 
some of the reviewed studies employed [44,49,59,62,63]. In other studies [61,62], authors employed exercise protocols based on previously validated tests [104,105], although few provide citations. Future studies may consider including repeatability data to provide better insight on how translatable the results are. Moreover, in publications without repeatability data (i.e., intra- and inter-individual variability), it is important to be cognizant of extrapolating the results beyond reasonable conclusions.

\subsection{Candidate Physiological Mechanisms}

To date, several physiological mechanisms have been reported to underpin $\mathrm{NO}_{3}{ }^{-}-$ induced performance enhancements, and the relative contribution of each mechanism may depend on the circumstance, participant population, and exercise protocol. Skeletal muscle function has been shown to increase in force production following 7 days of $\mathrm{NO}_{3}{ }^{-}$ supplementation in rodents, in which the authors attributed to increased protein expression in type II muscle fibers of dihydropyridine receptors and calsequestrin, as well as increased intracellular calcium concentration [28]. More recently, exercise tolerance was improved in rodents due to preserved calcium handling protein function [29]. However, in humans, Whitfield et al. [30] did not observe changes to any calcium handling protein content following 7 days of $\mathrm{NO}_{3}{ }^{-}$supplementation, which is in contrast to the rodent data $[28,29]$. However, since cysteine residues exist on many of the proteins that regulate excitationcontraction coupling [106], it could be possible that these proteins undergo the process of S-nitrosylation [19] in altering function during contraction without requiring any change in protein expression. Moreover, $\mathrm{NO}_{3}{ }^{-}$supplementation has been observed to modulate hydrogen peroxide [107], a reactive oxygen species molecule that has potential to alter force production independent from calcium via inducing signaling cascades [79]. Taken together, it remains a possibility that dietary $\mathrm{NO}_{3}{ }^{-}$supplementation evokes physiological effects and performance enhancements by modulating the regulatory processes of calcium release and reuptake and/or by altering skeletal muscle redox status.

Dietary $\mathrm{NO}_{3}{ }^{-}$supplementation has also been evidenced to influence the phosphocreatine system such that the phosphocreatine cost of energy production was lower and accompanied by an attenuated accumulation of metabolites during knee extensor exercise [31]. Although the exact mechanisms are unclear, by preserving phosphocreatine, it is possible that $\mathrm{NO}_{3}{ }^{-}$impacts the efficiency of this system and thus would benefit exercise protocols relying on the phosphocreatine system, such as explosive contractions. Furthermore, the blood flow distribution has been observed to improve between muscle fiber types following $\mathrm{NO}_{3}{ }^{-}$supplementation [32], which could suggest that recovery between high-intensity explosive repetitions may be improved and thus could preserve power across several repetitions. However, it is notable that $\mathrm{NO}_{3}{ }^{-}$supplementation has not been observed to impact phosphocreatine resynthesis unless in hypoxic conditions [108].

\section{Conclusions}

In conclusion, dietary $\mathrm{NO}_{3}{ }^{-}$supplementation may be effective in improving the power and velocity of explosive resistance exercise efforts such as weightlifting, as well as the total work, and sprint time of explosive repeated sprint-type exercise protocols in cycling and running. However, these effects may be dependent on the $\mathrm{NO}_{3}{ }^{-}$supplementation regimen, participant characteristics, and exercise modality. Furthermore, to improve the interpretation of results and to minimize false conclusions, future studies are encouraged to report the validity and reliability of the exercise protocols employed, given that relatively small differences may be observed. Clearly, more research is required to elucidate the impacts of manipulating dosing regimens in various exercise modalities and in females to understand how and when to administer $\mathrm{NO}_{3}{ }^{-}$supplementation for optimizing $\mathrm{NO}$ bioavailability to enhance explosive movements.

Author Contributions: Conceptualization, L.C. and R.D.; data curation, L.C., Á.L.-R. and R.D.; formal analysis, L.C., Á.L.-R., R.D. and R.T.; supervision, R.T. and R.D.; writing the original draft, 
L.C., R.T., Á.L.-R. and R.D.; edited and revised manuscript, R.T. and R.D. All authors have read and agreed to the published version of the manuscript.

Funding: This research received no external funding.

Institutional Review Board Statement: Not applicable.

Informed Consent Statement: Not applicable.

Data Availability Statement: All details can be requested from the corresponding author.

Conflicts of Interest: The authors declare no conflict of interest.

\section{References}

1. Stone, M.H.; Moir, G.; Glaister, M.; Sanders, R. How much strength is necessary? Phys. Ther. Sport 2002, 3, 88-96. [CrossRef]

2. Baker, J.S.; McCormick, M.C.; Robergs, R.A. Interaction among Skeletal Muscle Metabolic Energy Systems during Intense Exercise. J. Nutr. Metab. 2010, 2010, 905612. [CrossRef]

3. Cormie, P.; McGuigan, M.R.; Newton, R.U. Developing maximal neuromuscular power: Part 1-Biological basis of maximal power production. Sports Med. 2011, 41, 17-38. [CrossRef] [PubMed]

4. Baker, D.; Nance, S. The Relation Between Strength and Power in Professional Rugby League Players. J. Strength Cond. Res. 1999, 13, 224-229. [CrossRef]

5. Bigland-Ritchie, B.; Woods, J.J. Changes in muscle contractile properties and neural control during human muscular fatigue. Muscle Nerve 1984, 7, 691-699. [CrossRef] [PubMed]

6. Gibala, M.J.; Little, J.P.; MacDonald, M.J.; Hawley, J.A. Physiological adaptations to low-volume, high-intensity interval training in health and disease. J. Physiol. 2011, 2011. [CrossRef] [PubMed]

7. Baker, D.; Nance, S.; Moore, M. The load that maximizes the average mechanical power output during explosive bench press throws in highly trained athletes. J. Strength Cond. Res. 2001, 15, 20-24.

8. Maughan, R.J.; Burke, L.M.; Dvorak, J.; Larson-Meyer, D.E.; Peeling, P.; Phillips, S.M.; Rawson, E.S.; Walsh, N.P.; Garthe, I.; Geyer, H.; et al. IOC consensus statement: Dietary supplements and the high-performance athlete. Br. J. Sports Med. 2018, 52, 439-455. [CrossRef]

9. Hord, N.G.; Tang, Y.; Bryan, N.S. Food sources of nitrates and nitrites: The physiologic context for potential health benefits. Am. J. Clin. Nutr. 2009, 90, 1-10. [CrossRef]

10. Knapik, J.J.; Steelman, R.A.; Hoedebecke, S.S.; Austin, K.G.; Farina, E.K.; Lieberman, H.R. Prevalence of Dietary Supplement Use by Athletes: Systematic Review and Meta-Analysis. Sports Med. 2016, 46, 103-123. [CrossRef]

11. Wylie, L.J.; Kelly, J.; Bailey, S.J.; Blackwell, J.R.; Skiba, P.F.; Winyard, P.G.; Jeukendrup, A.E.; Vanhatalo, A.; Jones, A.M. Beetroot juice and exercise: Pharmacodynamic and dose-response relationships. J. Appl. Physiol. 2013, 115, 325-336. [CrossRef]

12. Gilchrist, M.; Winyard, P.G.; Benjamin, N. Dietary nitrate-Good or bad? Nitric Oxide 2010, 22, 104-109. [CrossRef]

13. Sacks, F.M.; Svetkey, L.P.; Vollmer, W.M.; Appel, L.J.; Bray, G.A.; Harsha, D.; Obarzanek, E.; Conlin, P.R.; Miller, E.R.; Simons-Morton, D.G.; et al. Effects on Blood Pressure of Reduced Dietary Sodium and the Dietary Approaches to Stop Hypertension (DASH) Diet. N. Engl. J. Med. 2001, 344, 3-10. [CrossRef] [PubMed]

14. Boorsma, R.K.; Whitfield, J.; Spriet, L.L. Beetroot Juice Supplementation Does Not Improve Performance of Elite 1500-m Runners. Med. Sci. Sports Exerc. 2014, 46, 2326-2334. [CrossRef] [PubMed]

15. Stamler, J.S.; Meissner, G. Physiology of nitric oxide in skeletal muscle. Physiol. Rev. 2001, 81, 209-237. [CrossRef] [PubMed]

16. Lundberg, J.O.; Weitzberg, E.; Gladwin, M.T. The nitrate-nitrite-nitric oxide pathway in physiology and therapeutics. Nat. Rev. Drug Discov. 2008, 7, 156-157. [CrossRef] [PubMed]

17. Spiegelhalder, B.; Eisenbrand, G.; Preussmann, R. Influence of dietary nitrate on nitrite content of human saliva: Possible relevance to in vivo formation of N-nitroso compounds. Food Cosmet. Toxicol. 1976, 14, 545-548. [CrossRef]

18. Benjamin, N.; O’Driscoll, F.; Dougall, H.; Duncan, C.; Smith, L.; Golden, M.; McKenzie, H. Stomach NO synthesis. Nature 1994, 368, 502. [CrossRef]

19. Hogg, N. The Biochemistry and Physiology of S-Nitrosothiols. Annu. Rev. Pharmacol. Toxicol. 2002, 42, 585-600. [CrossRef]

20. Modin, A.; Bjorne, H.; Herulf, M.; Alving, K.; Weitzberg, E.; Lundberg, J. Nitrite-derived nitric oxide: A possible mediator of 'acidic-metabolic' vasodilation. Acta Physiol. Scand. 2001, 171, 9-16. [CrossRef]

21. Castello, P.R.; David, P.S.; McClure, T.; Crook, Z.; Poyton, R.O. Mitochondrial cytochrome oxidase produces nitric oxide under hypoxic conditions: Implications for oxygen sensing and hypoxic signaling in eukaryotes. Cell Metab. 2006, 3, 277-287. [CrossRef] [PubMed]

22. Hlinský, T.; Kumstát, M.; Vajda, P. Effects of Dietary Nitrates on Time Trial Performance in Athletes with Different Training Status: Systematic Review. Nutrients 2020, 12, 2734. [CrossRef] [PubMed]

23. Rojas-Valverde, D.; Montoya-Rodríguez, J.; Azofeifa-Mora, C.; Sanchez-Urena, B. Effectiveness of beetroot juice derived nitrates supplementation on fatigue resistance during repeated-sprints: A systematic review. Crit. Rev. Food Sci. Nutr. 2020, 61, 3395-3406. [CrossRef] 
24. Juan, A.F.S.; Dominguez, R.; Lago-Rodríguez, Á.; Montoya, J.J.; Tan, R.; Bailey, S.J. Effects of Dietary Nitrate Supplementation on Weightlifting Exercise Performance in Healthy Adults: A Systematic Review. Nutrients 2020, 12, 2227. [CrossRef] [PubMed]

25. Senefeld, J.W.; Wiggins, C.C.; Regimbal, R.J.; Dominelli, P.B.; Baker, S.E.; Joyner, M.J. Ergogenic Effect of Nitrate Supplementation: A Systematic Review and Meta-analysis. Med. Sci. Sports Exerc. 2020, 52, 2250-2261. [CrossRef]

26. Domínguez, R.; Maté-Muñoz, J.L.; Cuenca, E.; García-Fernández, P.; Mata-Ordoñez, F.; Lozano-Estevan, M.C.; Veiga-Herreros, P.; da Silva, S.F.; Garnacho-Castaño, M.V. Effects of beetroot juice supplementation on intermittent high-intensity exercise efforts. J. Int. Soc. Sports Nutr. 2018, 15, 2. [CrossRef]

27. Calvo, J.L.; Alorda-Capo, F.; Pareja-Galeano, H.; Jiménez, S.L. Influence of Nitrate Supplementation on Endurance Cyclic Sports Performance: A Systematic Review. Nutrients 2020, 12, 1796. [CrossRef]

28. Hernández, A.; Schiffer, T.A.; Ivarsson, N.; Cheng, A.J.; Bruton, J.D.; Lundberg, J.O.; Weitzberg, E.; Westerblad, H. Dietary nitrate increases tetatnic [Ca2 ${ }^{+}$]i and contractile forcé in mouse fast-twitch muscle. J. Physiol. 2012, 590, 3575-3583. [CrossRef] [PubMed]

29. Bailey, S.J.; Gandra, P.G.; Jones, A.M.; Hogan, M.C.; Nogueira, L. Incubation with sodium nitrite attenuates fatigue development in intact single mouse fibres at physiological $\mathrm{PO}_{2}$. J. Physiol. 2019, 597, 5429-5443. [CrossRef]

30. Whitfield, J.; Gamu, D.; Heigenhauser, G.J.F.; van Loon, L.J.C.; Spriet, L.L.; Tupling, A.R.; Holloway, G.P. Beetroot Juice Increases Human Muscle Force without Changing Ca2+-Handling Proteins. Med. Sci. Sports Exerc. 2017, 49, 2016-2024. [CrossRef]

31. Bailey, S.J.; Fulford, J.; Vanhatalo, A.; Winyard, P.G.; Blackwell, J.R.; DiMenna, F.J.; Wilkerson, D.P.; Benjamin, N.; Jones, A.M. Dietary nitrate supplementation enhances muscle contractile efficiency during knee-extensor exercise in humans. J. Appl. Physiol. 2010, 109, 135-148. [CrossRef]

32. Ferguson, S.K.; Hirai, D.M.; Copp, S.W.; Holdsworth, C.T.; Allen, J.D.; Jones, A.M.; Musch, T.I.; Poole, D.C. Impact of dietary nitrate supplementation via beetroot juice on exercising muscle vascular control in rats. J. Physiol. 2013, 591, 547-557. [CrossRef] [PubMed]

33. Bailey, S.J.; Varnham, R.L.; DiMenna, F.J.; Breese, B.C.; Wylie, L.J.; Jones, A.M. Inorganic nitrate supplementation improves muscle oxygenation, $\mathrm{O}_{2}$ uptake kinetics, and exercise tolerance at high but not low pedal rates. J. Appl. Physiol. 2015, 118, 1396-1405. [CrossRef]

34. Breese, B.C.; McNarry, M.; Marwood, S.; Blackwell, J.R.; Bailey, S.; Jones, A.M. Beetroot juice supplementation speeds $\mathrm{O}_{2}$ uptake kinetics and improves exercise tolerance during severe-intensity exercise initiated from an elevated metabolic rate. Am. J. Physiol. Integr. Comp. Physiol. 2013, 305, R1441-R1450. [CrossRef] [PubMed]

35. Coggan, A.R.; Peterson, L. Dietary Nitrate Enhances the Contractile Properties of Human Skeletal Muscle. Exerc. Sport Sci. Rev. 2018, 46, 254-261. [CrossRef]

36. Wong, T.H.; Sim, A.; Burns, S.F. The Effect of Beetroot Ingestion on High-Intensity Interval Training: A Systematic Review and Meta-Analysis. Nutrients 2021, 13, 3674. [CrossRef]

37. Coggan, A.R.; Leibowitz, J.L.; Kadkhodayan, A.; Thomas, D.P.; Ramamurthy, S.; Spearie, C.A.; Waller, S.; Farmer, M.; Peterson, L. Effect of acute dietary nitrate intake on maximal knee extensor speed and power in healthy men and women. Nitric Oxide 2015, 48, 16-21. [CrossRef]

38. Coggan, A.R.; Leibowitz, J.L.; Spearie, C.A.; Kadkhodayan, A.; Thomas, D.P.; Ramamurthy, S.; Mahmood, K.; Park, S.; Waller, S.; Farmer, M.; et al. Acute Dietary Nitrate Intake Improves Muscle Contractile Function in Patients with Heart Failure: A Double-Blind, Placebo-Controlled, Randomized Trial. Circ. Hearth Fail 2015, 8, 914-920. [CrossRef]

39. Coggan, A.R.; Broadstreet, S.R.; Mikhalkova, D.; Bole, I.; Leibowitz, J.L.; Kadkhodayan, A.; Park, S.; Thomas, D.P.; Thies, D.; Peterson, L. Dietary nitrate-induced increases in human muscle power: High versus low responders. Physiol. Rep. 2018, 6, e13575. [CrossRef]

40. Jonvik, K.L.; Hoogervorst, D.; Peelen, H.B.; De Niet, M.; Verdijk, L.B.; Van Loon, L.J.C.; Van Dijk, J.-W. The impact of beetroot juice supplementation on muscular endurance, maximal strength and countermovement jump performance. Eur. J. Sport Sci. 2021, 21, 871-878. [CrossRef] [PubMed]

41. Cuenca, E.; Jodra, P.; Pérez-López, A.; González-Rodríguez, L.G.; Fernandes Da Silva, S.; Veiga-Herreros, P.; Domínguez, R. Effects of beetroot juice supplementation on performance and fatigue in a 30-s all-out sprint exercise: A randomized, double-blind cross-over study. Nutrients 2018, 10, 1222. [CrossRef]

42. Domínguez, R.; Cuenca, E.; Maté-Muñoz, J.L.; García-Fernández, P.; Serra-Paya, N.; Estevan, M.C.L.; Herreros, P.V.; Garnacho-Castaño, M.V. Effects of Beetroot Juice Supplementation on Cardiorespiratory Endurance in Athletes. A Systematic Review. Nutrients 2017, 9, 43. [CrossRef] [PubMed]

43. Jodra, P.; Domínguez, R.; Sanchez-Oliver, A.J.; Veiga-Herreros, P.; Bailey, S.J. Effect of Beetroot Juice Supplementation on Mood, Perceived Exertion, and Performance During a 30-Second Wingate Test. Int. J. Sports Physiol. Perform. 2020, 15, 243-248. [CrossRef] [PubMed]

44. Rimer, E.G.; Peterson, L.; Coggan, A.R.; Martin, J. Increase in Maximal Cycling Power With Acute Dietary Nitrate Supplementation. Int. J. Sports Physiol. Perform. 2016, 11, 715-720. [CrossRef]

45. Jonvik, K.; Nyakayiru, J.; Van Dijk, J.; Maase, K.; Ballak, S.; Senden, J.; van Loon, L.J.; Verdijk, L. Repeated-sprint performance and plasma responses following beetroot juice supplementation do not differ between recreational, competitive and elite sprint athletes. Eur. J. Sport Sci. 2018, 18, 524-533. [CrossRef]

46. Williams, T.D.; Martin, M.P.; Mintz, J.A.; Rogers, R.R.; Ballmann, C.G. Effect of Acute Beetroot Juice Supplementation on Bench Press Power, Velocity, and Repetition Volume. J. Strength Cond. Res. 2020, 34, 924-928. [CrossRef] 
47. Ranchal-Sanchez, A.; Diaz-Bernier, V.M.; Alonso De La Florida-Villagran, C.; Llorente-Cantarero, F.J.; Campos-Perez, J.; Jurado-Castro, J.M. Acute effects of beetroot juice supplements on resistance training: A randomized double-blind crossover. Nutrients 2020, 12, 1912. [CrossRef]

48. Henneman, E.; Somjen, G.; Carpenter, D.O. Excitability and Inhibitibility of Motoneurons of Different Sizes. J. Neurophysiol. 1965, 28, 599-620. [CrossRef]

49. Wylie, L.; Bailey, S.; Kelly, J.; Blackwell, J.R.; Vanhatalo, A.; Jones, A.M. Influence of beetroot juice supplementation on intermittent exercise performance. Eur. J. Appl. Physiol. 2016, 116, 415-425. [CrossRef]

50. Moher, D.; Liberati, A.; Tetzlaff, J.; Altman, D.G.; The PRISMA Group. Preferred reporting items for systematic reviews and meta-analyses: The PRISMA Statement. PLoS Med. 2009, 6, e1000097. [CrossRef] [PubMed]

51. Maher, C.G.; Sherrington, C.; Herbert, R.D.; Moseley, A.M.; Elkins, M. Reliability of the PEDro Scale for Rating Quality of Randomized Controlled Trials. Phys. Ther. 2003, 83, 713-721. [CrossRef]

52. Grgic, J. Caffeine ingestion enhances Wingate performance: A meta-analysis. Eur. J. Sport Sci. 2018, 18, 219-225. [CrossRef]

53. Van Tulder, M.; Furlan, A.; Bombardier, C.; Bouter, L. Updated Method Guidelines for Systematic Reviews in the Cochrane Collaboration Back Review Group. Spine 2003, 28, 1290-1299. [CrossRef]

54. McCrary, J.M.; Ackermann, B.; Halaki, M. A systematic review of the effects of upper body warm-up on performance and injury. Br. J. Sports Med. 2015, 49, 935-942. [CrossRef]

55. Cohen, J. Statistical Power Analysis for the Behavioral Sciences, 2nd ed.; Routledge Academic: New York, NY, USA, 1988.

56. Kokkinoplitis, K.; Chester, N. The effect of beetroot juice on repeated sprint performance and muscle force production. J. Phys. Educ. Sport 2014, 14, 242-247.

57. López-Samanes, Á.; Pérez-López, A.; Moreno-Pérez, V.; Nakamura, F.Y.; Acebes-Sánchez, J.; Quintana-Milla, I.; Sánchez-Oliver, A.J.; Moreno-Pérez, D.; Fernández-Elías, V.E.; Domínguez, R. Effects of Beetroot Juice Ingestion on Physical Performance in Highly Competitive Tennis Players. Nutrients 2020, 12, 584. [CrossRef] [PubMed]

58. Shamseer, L.; Moher, D.; Clarke, M.; Ghersi, D.; Liberati, A.; Petticrew, M.; Shekelle, P.; Stewart, L.A.; The PRISMA-P Group Preferred reporting items for systematic review and meta-analysis protocols (PRISMA-P) 2015: Elaboration and explanation. BMJ 2015, 349, g7647. [CrossRef] [PubMed]

59. Thompson, C.; Wylie, L.J.; Fulford, J.; Kelly, J.; Black, M.I.; McDonagh, S.T.; Jeukendrup, A.E.; Vanhatalo, A.; Jones, A.M. Dietary nitrate improves sprint performance and cognitive function during prolonged intermittent exercise. Eur. J. Appl. Physiol. 2015, 115, 1825-1834. [CrossRef] [PubMed]

60. Thompson, C.; Vanhatalo, A.; Jell, H.; Fulford, J.; Carter, J.; Nyman, L.; Bailey, S.J.; Jones, A.M. Dietary nitrate supplementation improves sprint and high-intensity intermittent running performance. Nitric Oxide 2016, 61, 55-61. [CrossRef]

61. Buck, C.L.; Henry, T.; Guelfi, K.; Dawson, B.; Mc Naughton, L.R.; Wallman, K. Effects of sodium phosphate and beetroot juice supplementation on repeated-sprint ability in females. Eur. J. Appl. Physiol. 2015, 115, 2205-2213. [CrossRef]

62. Smith, K.; Muggeridge, D.J.; Easton, C.; Ross, M.D. An acute dose of inorganic dietary nitrate does not improve high-intensity, intermittent exercise performance in temperate or hot and humid conditions. Graefe's Arch. Clin. Exp. Ophthalmol. 2019, 119, 723-733. [CrossRef] [PubMed]

63. Kent, G.L.; Dawson, B.; McNaughton, L.R.; Cox, G.; Burke, L.M.; Peeling, P. The effect of beetroot juice supplementation on repeat-sprint performance in hypoxia. J. Sports Sci. 2019, 37, 339-346. [CrossRef] [PubMed]

64. Clifford, T.; Berntzen, B.; Davison, G.W.; West, D.J.; Howatson, G.; Stevenson, E.J. Effects of Beetroot Juice on Recovery of Muscle Function and Performance between Bouts of Repeated Sprint Exercise. Nutrients 2016, 8, 506. [CrossRef]

65. Rodríguez, A.; Castillo, D.; Raya-González, J.; Domínguez, R.; Bailey, S.J. Beetroot juice supplementation increases concentric and eccentric muscle power output. Original investigation. J. Sci. Med. Sport 2020, 24, 80-84. [CrossRef] [PubMed]

66. Tillin, N.A.; Moudy, S.; Nourse, K.M.; Tyler, C.J. Nitrate Supplement Benefits Contractile Forces in Fatigued but Not Unfatigued Muscle. Med. Sci. Sports Exerc. 2018, 50, 2122-2131. [CrossRef] [PubMed]

67. Kramer, S.J.; Baur, D.A.; Spicer, M.T.; Vukovich, M.; Ormsbee, M.J. The effect of six days of dietary nitrate supplementation on performance in trained CrossFit athletes. J. Int. Soc. Sports Nutr. 2016, 13, 39. [CrossRef]

68. Haider, G.; Folland, J.P. Nitrate Supplementation Enhances the Contractile Properties of Human Skeletal Muscle. Med. Sci. Sports Exerc. 2014, 46, 2234-2243. [CrossRef] [PubMed]

69. Porcelli, S.; Ramaglia, M.; Bellistri, G.; Pavei, G.; Pugliese, L.; Montorsi, M.; Rasica, L.; Marzorati, M. Aerobic Fitness Affects the Exercise Performance Responses to Nitrate Supplementation. Med. Sci. Sports Exerc. 2015, 47, 1643-1651. [CrossRef]

70. Wilkerson, D.P.; Hayward, G.M.; Bailey, S.J.; Vanhatalo, A.; Blackwell, J.R.; Jones, A.M. Influence of acute dietary nitrate supplementation on 50 mile time trial performance in well-trained cyclists. Graefe's Arch. Clin. Exp. Ophthalmol. 2012, 112, 4127-4134. [CrossRef]

71. Bond, H.; Morton, L.; Braakhuis, A. Dietary Nitrate Supplementation Improves Rowing Performance in Well-Trained Rowers. Int. J. Sport Nutr. Exerc. Metab. 2012, 22, 251-256. [CrossRef]

72. Trappe, S.; Luden, N.; Minchev, K.; Raue, U.; Jemiolo, B.; Trappe, T.A. Skeletal muscle signature of a champion sprint runner. J. Appl. Physiol. 2015, 118, 1460-1466. [CrossRef] [PubMed]

73. Wilson, J.M.; Loenneke, J.P.; Jo, E.; Wilson, G.J.; Zourdos, M.C.; Kim, J.-S. The Effects of Endurance, Strength, and Power Training on Muscle Fiber Type Shifting. J. Strength Cond. Res. 2012, 26, 1724-1729. [CrossRef] [PubMed] 
74. Rodríguez-Rosell, D.; Blanco, F.P.; Aagaard, P.; González-Badillo, J.J. Physiological and methodological aspects of rate of force development assessment in human skeletal muscle. Clin. Physiol. Funct. Imaging 2018, 38, 743-762. [CrossRef] [PubMed]

75. Wootton-Beard, P.C.; Ryan, L. Combined use of Multiple Methodologies for the Measurement of Total Antioxidant Capacity in UK Commercially Available Vegetable Juices. Plant Foods Hum. Nutr. 2012, 67, 142-147. [CrossRef]

76. Gago, B.; Lundberg, J.O.; Barbosa, R.M.; Laranjinha, J. Red wine-dependent reduction of nitrite to nitric oxide in the stomach. Free Radic. Biol. Med. 2007, 43, 1233-1242. [CrossRef] [PubMed]

77. Flueck, J.L.; Bogdanova, A.; Mettler, S.; Perret, C. Is beetroot juice more effective than sodium nitrate? The effects of equimolar nitrate dosages of nitrate-rich beetroot juice and sodium nitrate on oxygen consumption during exercise. Appl. Physiol. Nutr. Metab. 2016, 41, 421-429. [CrossRef]

78. Pawlak-Chaouch, M.; Boissière, J.; Gamelin, F.X.; Cuvelier, G.; Berthoin, S.; Aucouturier, J. Effect of dietary nitrate supplementation on metabolic rate during rest and exercise in human: A systematic review and meta-analysis. Nitric Oxide 2016, 53, 65-76. [CrossRef]

79. Reid, M.B. Redox interventions to increase exercise performance. J. Physiol. 2015, 594, 5125-5133. [CrossRef]

80. Jonvik, K.L.; Nyakayiru, J.; Van Dijk, J.-W.; Wardenaar, F.C.; Van Loon, L.J.; Verdijk, L.B. Habitual Dietary Nitrate Intake in Highly Trained Athletes. Int. J. Sport Nutr. Exerc. Metab. 2017, 27, 148-157. [CrossRef]

81. Glaister, M.; Pattison, J.R.; Muniz-Pumares, D.; Patterson, S.; Foley, P. Effects of Dietary Nitrate, Caffeine, and Their Combination on 20-km Cycling Time Trial Performance. J. Strength Cond. Res. 2015, 29, 165-174. [CrossRef]

82. Karampelas, D.; Antonopoulos, K.; Michailidis, Y.; Mitrotasios, M.; Mandroukas, A.; Metaxas, T. Comparison of Ergogenic Effects of Caffeine and Nitrate Supplementation on Speed, Power and Repeated Sprint Performance of Soccer Players. Physiologia 2021, 1, 2. [CrossRef]

83. Lane, S.C.; Hawley, J.A.; Desbrow, B.; Jones, A.M.; Blackwell, J.R.; Ross, M.L.; Zemski, A.J.; Burke, L.M. Single and combined effects of beetroot juice and caffeine supplementation on cycling time trial performance. Appl. Physiol. Nutr. Metab. 2014, 39, 1050-1057. [CrossRef]

84. Callahan, M.J.; Parr, E.; Hawley, J.; Burke, L.M. Single and Combined Effects of Beetroot Crystals and Sodium Bicarbonate on 4-km Cycling Time Trial Performance. Int. J. Sport Nutr. Exerc. Metab. 2017, 27, 271-278. [CrossRef]

85. Nyakayiru, J.; van Loon, L.J.; Verdijk, L.B. Could intramuscular storage of dietary nitrate contribute to its ergogenic effect? A mini-review. Free Radic. Biol. Med. 2020, 152, 295-300. [CrossRef]

86. Gilliard, C.N.; Lam, J.K.; Cassel, K.S.; Park, J.W.; Schechter, A.N.; Piknova, B. Effect of dietary nitrate levels on nitrate fluxes in rat skeletal muscle and liver. Nitric Oxide 2018, 75, 1-7. [CrossRef] [PubMed]

87. Piknova, B.; Park, J.W.; Swanson, K.M.; Dey, S.; Noguchi, C.T.; Schechter, A.N. Skeletal muscle as an endogenous nitrate reservoir. Nitric Oxide 2015, 47, 10-16. [CrossRef]

88. Piknova, B.; Park, J.W.; Lam, K.K.J.; Schechter, A.N. Nitrate as a source of nitrite and nitric oxide during exercise hyperemia in rat skeletal muscle. Nitric Oxide 2016, 55-56, 54-61. [CrossRef] [PubMed]

89. Wylie, L.J.; Park, J.W.; Vanhatalo, A.; Kadach, S.; Black, M.I.; Stoyanov, Z.; Schechter, A.N.; Jones, A.M.; Piknova, B. Human skeletal muscle nitrate store: Influence of dietary nitrate supplementation and exercise. J. Physiol. 2019, 597, 5565-5576. [CrossRef] [PubMed]

90. Hyde, E.R.; Andrade, F.; Vaksman, Z.; Parthasarathy, K.; Jiang, H.; Parthasarathy, D.K.; Torregrossa, A.C.; Tribble, G.; Kaplan, H.B.; Petrosino, J.F.; et al. Metagenomic Analysis of Nitrate-Reducing Bacteria in the Oral Cavity: Implications for Nitric Oxide Homeostasis. PLoS ONE 2014, 9, e88645. [CrossRef]

91. Govoni, M.; Jansson, E.Å.; Weitzberg, E.; Lundberg, J.O. The increase in plasma nitrite after a dietary nitrate load is markedly attenuated by an antibacterial mouthwash. Nitric Oxide 2008, 19, 333-337. [CrossRef] [PubMed]

92. Petersson, J.; Carlström, M.; Schreiber, O.; Phillipson, M.; Christoffersson, G.; Jägare, A.; Roos, S.; Jansson, E.Å.; Persson, A.E.G.; Lundberg, J.O.; et al. Gastroprotective and blood pressure lowering effects of dietary nitrate are abolished by an antiseptic mouthwash. Free Radic. Biol. Med. 2009, 46, 1068-1075. [CrossRef]

93. Bailey, S.J.; Blackwell, J.R.; Wylie, L.J.; Holland, T.; Winyard, P.G.; Jones, A.M. Improvement in blood pressure after short-term inorganic nitrate supplementation is attenuated in cigarette smokers compared to non-smoking controls. Nitric Oxide 2016, 61, 29-37. [CrossRef] [PubMed]

94. Vanhatalo, A.; L'Heureux, J.E.; Kelly, J.; Blackwell, J.R.; Wylie, L.J.; Fulford, J.; Winyard, P.G.; Williams, D.W.; van der Giezen, M.; Jones, A.M. Network analysis of nitrate-sensitive oral microbiome reveals interactions with cognitive function and cardiovascular health across dietary interventions. Redox Biol. 2021, 41, 101933. [CrossRef] [PubMed]

95. Costello, J.; Bieuzen, F.; Bleakley, C. Where are all the female participants in Sports and Exercise Medicine research? Eur. J. Sport Sci. 2014, 14, 847-851. [CrossRef] [PubMed]

96. Wickham, K.A.; Spriet, L.L. No longer beeting around the bush: A review of potential sex differences with dietary nitrate supplementation. Appl. Physiol. Nutr. Metab. 2019, 44, 915-924. [CrossRef] [PubMed]

97. Baranauskas, M.N.; Freemas, J.A.; Tan, R.; Carter, S.J. Moving beyond inclusion: Methodological considerations for the menstrual cycle and menopause in research evaluating effects of dietary nitrate on vascular function. Nitric Oxide 2021, 118, 39-48. [CrossRef] [PubMed] 
98. Kapil, V.; Rathod, K.S.; Khambata, R.S.; Bahra, M.; Velmurugan, S.; Purba, A.; Watson, D.S.; Barnes, M.R.; Wade, W.G.; Ahluwalia, A. Sex differences in the nitrate-nitrite-NO• pathway: Role of oral nitrate-reducing bacteria. Free Radic. Biol. Med. 2018, 126, 113-121. [CrossRef] [PubMed]

99. Mendez-Villanueva, A.; Bishop, D.; Hamer, P. Reproducibility of a 6-s maximal cycling sprint test. J. Sci. Med. Sport 2007, 10, 323-326. [CrossRef]

100. Bishop, D.; Spencer, M.; Duffield, R.; Lawrence, S. The validity of a repeated spring ability test. J. Sci. Med. Sport 2001, 4, 19-29. [CrossRef]

101. Glaister, M.; Stone, M.H.; Stewart, A.M.; Hughes, M.; Moir, G.L. The reliability and validity of fatigue measures during short-duration maximal-intensity intermittent cycling. J. Strength Cond. Res. 2004, 18, 459-462.

102. Sim, A.Y.; Dawson, B.T.; Guelfi, K.J.; E Wallman, K.; Young, W.B. Effects of Static Stretching in Warm-Up on Repeated Sprint Performance. J. Strength Cond. Res. 2009, 23, 2155-2162. [CrossRef] [PubMed]

103. Darrall-Jones, J.D.; Jones, B.; Roe, G.; Till, K. Reliability and Usefulness of Linear Sprint Testing in Adolescent Rugby Union and League Players. J. Strength Cond. Res. 2016, 30, 1359-1364. [CrossRef] [PubMed]

104. Spencer, M.; Lawrence, S.; Rechichi, C.; Bishop, D.; Dawson, B.; Goodman, C. Time-motion analysis of elite field hockey, with special reference to repeated-sprint activity. J. Sports Sci. 2004, 22, 843-850. [CrossRef]

105. Spencer, M.; Bishop, D.; Dawson, B.; Goodman, C. Physiological and Metabolic Responses of Repeated-Sprint Activities. Sports Med. 2005, 35, 1025-1044. [CrossRef] [PubMed]

106. Stamler, J.S. Redox signaling: Nitrosylation and related target interactions of nitric oxide. Cell 1994, 78, 931-936. [CrossRef]

107. Whitfield, J.; Ludzki, A.; Heigenhauser, G.J.F.; Senden, J.M.G.; Verdijk, L.; van Loon, L.J.; Spriet, L.L.; Holloway, G.P. Beetroot juice supplementation reduces whole body oxygen consumption but does not improve indices of mitochondrial efficiency in human skeletal muscle. J. Physiol. 2016, 594, 421-435. [CrossRef]

108. Vanhatalo, A.; Jones, A.M.; Blackwell, J.R.; Winyard, P.G.; Fulford, J. Dietary nitrate accelerates postexercise muscle metabolic recovery and $\mathrm{O}_{2}$ delivery in hpoxia. J. Appl. Physiol. 2014, 117, 1460-1470. [CrossRef] 